Pharmaceutics

International Journal of

Manuscript Draft

Manuscript Number: IJP-D-20-01159

Title: Cyclodextrin Nanoparticle Bound Oral Camptothecin for Colorectal Cancer: Formulation Development and Optimization

Article Type: VSI: T. Loftsson 70th Birthday

Section/Category: Pharmaceutical Nanotechnology

Keywords: Colorectal Cancer; Oral Administration; Nanoparticle; Camptothecin; Cyclodextrin

Abstract: Colorectal cancer (CRC) is currently the third most lethal cancer globally. Chemotherapeutic drugs used for CRC are administered as intravenous (iv) infusion due to their low oral bioavailability resulting from their unfavorable physicochemical properties. Non-selective biodistribution and difficulties of parenteral administration reduce patient compliance and treatment efficacy. To develop cyclodextrin (CD) based cationic nanoparticles (NPS) for CRC treatment with model drug camptothecin (CPT) that can be administered orally, protecting CPT through gastrointestinal tract (GIT), accumulating at mucus layer and providing an effective local treatment for the tumor area. NPs using two different amphiphilic CDs were prepared and coated with polyethylenimine (PEI) or chitosan (CS) to obtain positively charged surface for all formulations. Pre-formulation studies resulted in optimal formulation, CPT loaded Poly- $-C D-C 6$ NPs, with $135 \mathrm{~nm}$ diameter and zeta potential of +40 mV. In vitro release study was designed to represent gastrointestinal $\mathrm{pH}$ and transit time revealing 52\% of encapsulated CPT successfully delivered all the way to simulated colon. CPT bound to Poly- $\beta-C D-C 6$ NPs exhibited higher cytotoxicity on HT-29 cells compared to equivalent CPT in solution. Caco-2 cell permeability studies showed 276\% increase in CPT permeability and significantly higher mucosal penetration in cationic CD nanoparticle form. 


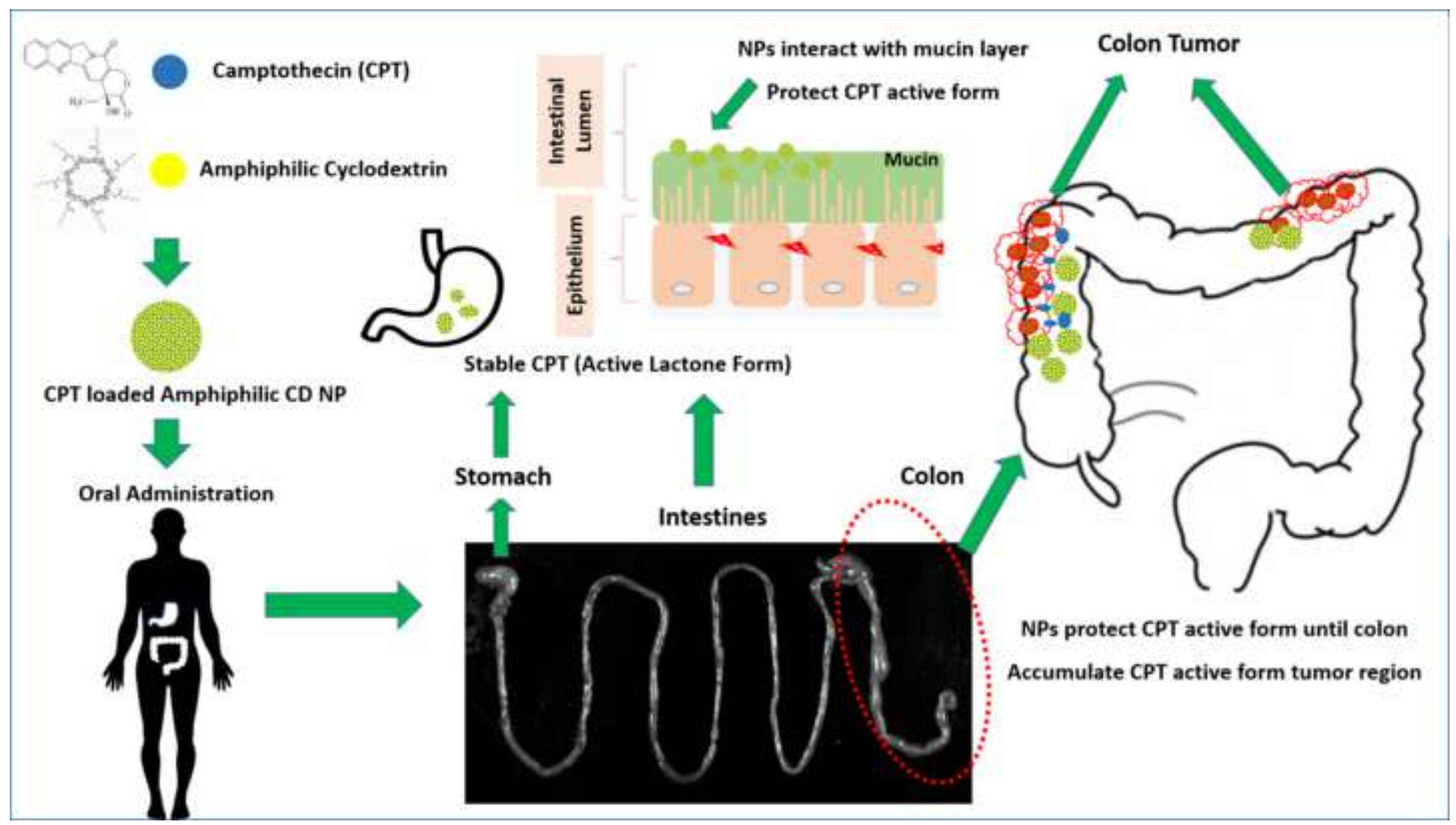




\title{
Cyclodextrin Nanoparticle Bound Oral Camptothecin for Colorectal Cancer: Formulation Development and Optimization
}

\author{
Sedat ÜNAL ${ }^{1,2}$, Yeşim AKTAŞ ${ }^{1}$, Juan M. Benito ${ }^{3}$, Erem BiLENSOY ${ }^{2}$ \\ 1. Department of Pharmaceutical Technology, Faculty of Pharmacy, Erciyes University, Kayseri, 38280, Turkey \\ 2. Department of Pharmaceutical Technology, Faculty of Pharmacy, Hacettepe University, Ankara, 06100, \\ Turkey \\ 3. Institute for Chemical Research, CSIC - University of Sevilla, Av. Americo Vespucio 49, Sevilla, 41092, Spain
}

\begin{abstract}
Colorectal cancer (CRC) is currently the third most lethal cancer globally. Chemotherapeutic drugs used for CRC are administered as intravenous (iv) infusion due to their low oral bioavailability resulting from their unfavorable physicochemical properties. Non-selective biodistribution and difficulties of parenteral administration reduce patient compliance and treatment efficacy. To develop cyclodextrin (CD) based cationic nanoparticles (NPS) for CRC treatment with model drug camptothecin (CPT) that can be administered orally, protecting CPT through gastrointestinal tract (GIT), accumulating at mucus layer and providing an effective local treatment for the tumor area. NPs using two different amphiphilic CDs were prepared and coated with polyethylenimine (PEI) or chitosan (CS) to obtain positively charged surface for all formulations. Pre-formulation studies resulted in optimal formulation, CPT loaded Poly- $\beta-C D-C 6$ NPs, with $135 \mathrm{~nm}$ diameter and zeta potential of $+40 \mathrm{mV}$. In vitro release study was designed to represent gastrointestinal $\mathrm{pH}$ and transit time revealing 52\% of encapsulated CPT successfully delivered all the way to simulated colon. CPT bound to Poly- $\beta$-CD-C6 NPs exhibited higher cytotoxicity on HT-29 cells compared to equivalent CPT in solution. Caco-2 cell permeability studies showed $276 \%$ increase in CPT permeability and significantly higher mucosal penetration in cationic CD nanoparticle form.
\end{abstract}

Keywords: Colorectal Cancer, Oral Administration, Nanoparticle, Camptothecin, Cyclodextrin 


\section{Graphical Abstract}

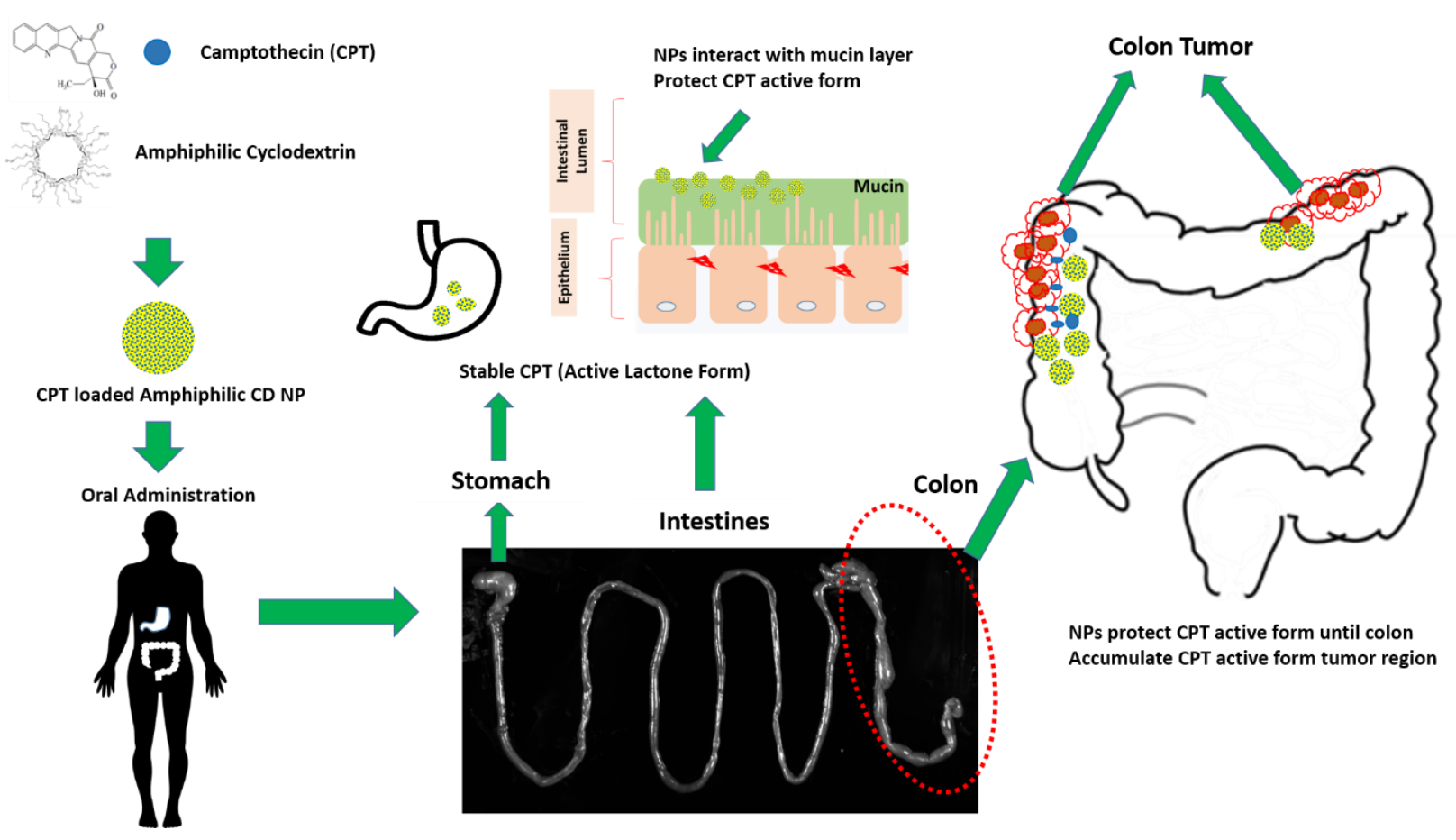

\section{Introduction}

Cancer is one of the most common chronic diseases in the world, characterized by the uncontrolled proliferation and spread of cells (Bilensoy, 2010). Cancer is a complex process that results in increased activity of cellular oncogene functions and decreased activity in tumor suppressor genes. Since cancers can be caused by any of the different cell types in the body, they can vary depending on the cell type from which they originate and subsequently the formation process and the responses to treatment varies (Jones and Baylin, 2007). Colorectal cancers (CRC) develop as polyps, usually on the inner wall of the colon epithelium, showing abnormal growth and proliferation as a malignant neoplasm in the mucosa of the colon and rectum. Polyp-related tumors that develop and grow in the inner colon epithelium tend to metastasize to other organs by invading muscle tissues and lymph nodes. Tumor recurrence and distant metastasis tendencies are the two most important factors affecting survival in patients with colorectal cancer. Despite diagnostic and therapeutic advances, studies are still underway to seek new diagnosis and treatment approaches as it is associated with high morbidity and mortality and the lack of an effective 
oral chemotherapy is one of the most important challenges in cancer treatment in the world (You et al., 2016).

Oral drug administration is the most preferred route because of the ease of administration especially for chronic patients who receive long-term medication (Date et al., 2016). It is less stressful and cost effective for the patient since it is painless and selfadministered without any need for a health facility or health professional for each dosing (Plapied et al., 2011). Orally administered drugs can be designed to treat gastrointestinal tract (GIT) diseases locally or systemically. Despite the advantages provided by oral drug administration, there are some physiological barriers that should be overcome from a biopharmaceutical point of view. These barriers can be physiological or biochemical barriers characteristic of GIT, but also can be caused by physicochemical properties of the drug such as solubility or stability. Camptothecin is a typical example of an anticancer molecule with no clinical efficacy due to its solubility and stability issues under physiological conditions.

Camptothecin (CPT) is an antineoplastic agent with topoisomerase I inhibitor activity. Topoisomerases are enzymes involved in the vital functions of DNA, such as DNA replication and transcription. Due to the pentacyclic alkaloid inherent in its structure, CPT can show strong anticancer activity in a broad spectrum of cancers such as metastatic colon cancers, small cell lung cancers, ovarian cancers, breast cancers (Zunino et al., 2002). CPT is known to have two different forms that are lactone and carboxylate. The ring $(E)$ in the active lactone form is opened and converted to the carboxylate form through hydrolysis. The conversion between forms is $\mathrm{pH}$ dependent and reversible, rapidly changing to the carboxylate form at the alkaline or physiological $\mathrm{pH}$, resulting in decreased clinical efficiency and increased toxicity. In this context, research is ongoing to develop innovative CPT formulations to overcome existing obstacles and to enable clinical use of CPT (Chao et al., 2017; Davis, 2009). The lactone form in the CPT structure, which has not transitioned to the carboxylate form, is the functional part that is primarily responsible for anticancer activity because only the CPT in the lactone form can pass through the cellular membranes and exhibit topoisomerase I inhibitory activity. Because of this situation, CPT shows serious stability and activity problems in physiological environment, and also shows low bioavailability due to low permeability, P-glycoprotein efflux pump system effect and water solubility problem in oral administration (Huang et al., 2008). 
Nanoparticle-bound drug delivery has some advantages compared to conventional drug delivery systems. Selection of the nanoparticle material type and modification of particle size and surface properties not only allow modulation in drug loading and release profile but also render targeting ability and better stability at the site of action, subsequently resulting in an increase in the therapeutic response and reduction off-target adverse effects (Mohanraj and Chen, 2006). It is possible to overcome the GIT-related multiple barriers both because the drug delivery system with oral administration of nanoparticulate drug delivery systems. The nanoparticles allow the encapsulated drug to be protected from chemical or enzymatic degradation and to control the release profile and release site of the active ingredient from the drug delivery system. Thus, treatment can become more effective and applied dose, dosing frequency and possible side effects can be reduced and as a consequence patients compliance can be increased (Luo et al., 2014).

Cyclodextrins (CD) are natural cyclic oligosaccharide structure obtained by enzymatic degradation of starch with glucosyltransferase. The internal cavity of CDs, that have a threedimensional morphology described as cone-like, is hydrophobic and the external structure is hydrophilic; which allows the formation of water-soluble complexes including drugs which exhibit poor water solubility in the apolar cavity of the cyclodextrins (Trapani et al., 2010). Thus, with the drug-cyclodextrin inclusion complex formation, complexed drug can be protected from enzymatic or hydrolytic effects in physiological environment resulting in higher bioavailability due to increased stability and improved solubility (Qiu et al., 2017).

Amphiphilic CDs are synthesized in order to overcome the limitations of natural cyclodextrins in the pharmaceutical field by facial-selective chemical derivatization methodologies. Thus, selectively-grafting bonding linear or branched aliphatic chains of different lengths and/or structures on either the primary and/or secondary faces of natural cyclodextrins (mostly $\alpha$ and $\beta-C D$ ) impart cyclodextrins self-assembling abilities that eventually form nanoscale carriers in an organic solvent: water interface spontaneously without any need for auxiliary solvent and/or surfactant (Erdogar et al., 2017). They are able to encapsulate hydrophobic drugs both in their cavity, between the aliphatic chains lining the primary or secondary face and within the matrix structure that several amphiphilic CDs form as supramolecular aggregate in the form of a nanoparticle (Bilensoy and Hincal, 2009). 
In this study, amphiphilic cyclodextrin nanocarriers were prepared using non-ionic 6-0CAPRO- $\beta-C D$, with a negative surface charge in aqueous solutions and the polycationic amphiphilic $\beta-C D$ derivative Poly- $\beta-C D-C 6$, originally developed for complexation and delivery of nucleic acids (Díaz-Moscoso et al., 2008; Díaz-Moscoso et al., 2009; Jiménez Blanco et al. 2017). 6-O-CAPRO- $\beta$-CD nanoparticles were coated with either positively charged polyethyleneimine (PEI) or chitosan (CS) polymers in order to impart a positive surface charge to the nanoparticles. Cyclodextrins are used for drug targeting to the colon, because they are not absorbed or degraded in the stomach or intestines and deliver the guest/encapsulated molecule to the colon to release the active substance in the colon region after being dissociated by the colonic microflora. The purpose of this study is to develop a new and unique approach for colon drug targeting for localized treatment of colon tumors. The amphiphilic cyclodextrin self-assembled nanoparticle material is intended to remain intact along the upper segments of the GIT, then dissociated by the colonic microflora, facilitating the local release of the chemotherapeutic drug at the colon region and provide an effective and safe nanomedicine for oral administration. Camptothecin was incorporated as model chemotherapeutic molecule with severe stability and bioavailability problems.

\section{Material and methods}

\section{Material}

6-O-CAPRO- $\beta$-CD (Mw=1813 g/mol) and Poly- $\beta-C D-C 6 ~(M w=3178.15 \mathrm{~g} / \mathrm{mol}$ ) (Díaz-Moscoso et al., 2008) seen in Figure 1. were synthetized, purified and characterized in Institute for Chemical Research (CSIC-University of Sevilla, Spain) (Varan et al., 2016). 

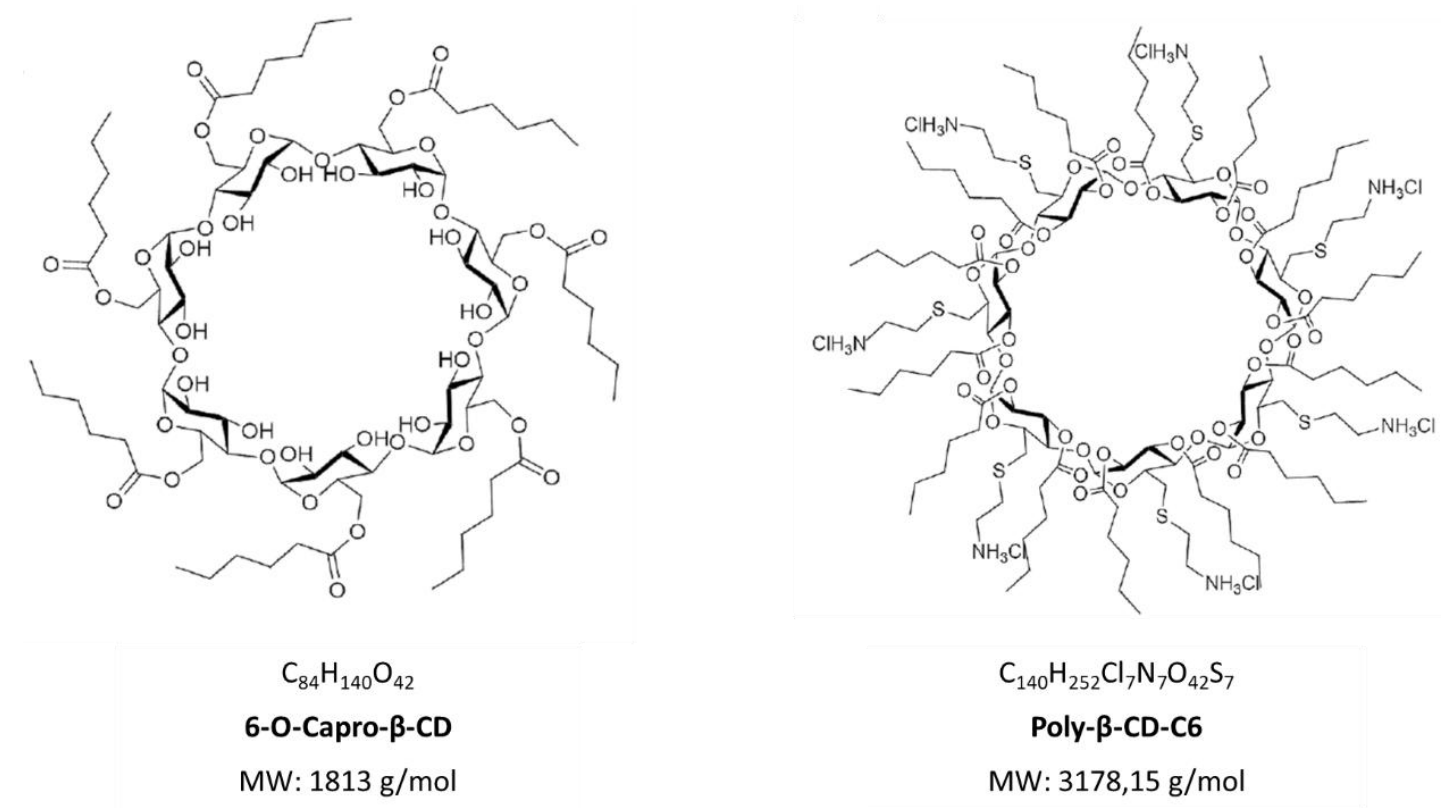

Figure 1. Schematic representation and physicochemical properties of $6-0-C A P R O-\beta-C D$ and Poly- $\beta-C D-C 6$.

20-(S)-(+)-camptothecin (95 \% HPLC powder, MW: $348.35 \mathrm{~g} / \mathrm{mol}$ ), polyethylenimine (branched, Mw: 25,000), dialysis cellulose tubing membrane (average flat width $25 \mathrm{~mm}$, MWCO: 14,000 Da), gelatin Type B from bovine skin, mucine from porcine stomach (Type II), DTPA (diethylenetriaminepentaacetic acid, min 99\%, titration) and egg yolk emulsion were purchased from Sigma\&Aldrich, USA. Chitosan (Protasan UP G-113; Mw: <200 kDa) was purchased from Novamatrix, Norway. All other chemicals used were of analytical grade and obtained from Sigma\&Aldrich.

\section{Methods}

\section{Preparation of blank and CPT-loaded amphiphilic CD nanoparticles}

All nanoparticles were prepared by nanoprecipitation method as described previously for amphiphilic CD nanoparticles (Varan et al., 2017a). Briefly, variable amounts of 6-O-CAPRO$\beta-C D$ or Poly- $\beta-C D-C 6$ were dissolved in $1 \mathrm{~mL}$ of organic solvent (ethanol or acetone) to yield a final concentration of $0.05,0.1,0.5 \%(\mathrm{w} / \mathrm{v})$ as organic phase. This organic phase was added dropwise into water phase consisting only of ultrapure water $(2 \mathrm{~mL})$ under magnetic stirring (30 $\mathrm{min}$ ) at room temperature. Then the organic solvent was evaporated with rotary evaporator at $45{ }^{\circ} \mathrm{C}$ to the desired final volume of $2 \mathrm{~mL}$. To prepare CS or PEI coated 6-O- 
CAPRO- $\beta-C D$ nanoparticles, same technique was run in the presence of $C S(0.01,0.025,0.05$ $\%(\mathrm{w} / \mathrm{v}))$ or PEI $(2.5,5,10 \%(\mathrm{w} / \mathrm{v}))$ dissolved in the aqueous phase. Pre-formulation studies to optimize nanoparticle formulations with desired particle size, surface charge and physical stability parameters should be applied as follows; organic phase: aqueous phase ratio 1:2 $(\mathrm{v} / \mathrm{v})$, concentration of amphiphilic cyclodextrin at $0.1 \%(\mathrm{w} / \mathrm{v})$, CS (Protasan UP G-113) concentration at $0.025 \%(\mathrm{w} / \mathrm{v})$ and the PEI (polyethyleneimine) concentration at $5 \%(\mathrm{w} / \mathrm{v})$. To obtain the camptothecin-loaded nanoparticles, CPT (10\% of CD weight) was added and dissolved in organic phase and the same technique described previously was employed to form drug loaded nanoparticles dispersion spontaneously.

\section{In vitro Characterization of the Nanoparticles}

\section{Mean Particle Size and Surface Charge}

The mean particle size $(\mathrm{nm})$, polydispersity index (PDI) and zeta potential (mV) of cationic amphiphilic CD nanoparticles were determined by dynamic light scattering (DLS) (Malvern Zetasizer Nano ZS series, UK) with a disposable capillary cell. All measurements were performed at an angle of $173^{\circ}$ for particle size and $12.8^{\circ}$ for zeta potential. All formulations were measured at room temperature and carried out in triplicate. Particle size distribution was calculated as mean diameter $(\mathrm{nm}) \pm$ standard deviation (SD) and PDI. Zeta potential $(\mathrm{mV})$ was stated as the average of three subsequent measurements \pm SD.

\section{Determination of drug loading capacity}

The amounts of CPT in the lactone and carboxylate forms in amphiphilic CD nanoparticle formulations were measured directly by a validated isocratic HPLC method with a fluorescence detector (HP Agilent 1100, Germany). HPLC method was monitored using 435 and $370 \mathrm{~nm}$ (emission and excitation wavelengths), respectively (Warner and Burke, 1997). Briefly, after preparation of nanoparticles, separation of precipitated excess CD and undissolved or free drug was carried out by centrifugation at $3500 \mathrm{rpm}$ for $15 \mathrm{~min}$. Supernatant containing CPT-loaded amphiphilic CD nanoparticle dispersion was lyophilized for 24 hours to obtain nanoparticle powder. Lyophilized nanoparticle powder was dissolved in a mixture of DCM: DMSO $(1: 1 \mathrm{v} / \mathrm{v})$ to quantify total nanoparticle bound CPT according to the equation stated below. 
Nanoparticle bound drug \% = Calculated drug amount (mcg) / Initial drug added to organic phase $(\mathrm{mcg}) \times 100$

\section{In vitro CPT release from amphiphilic CD nanoparticles}

In vitro release characteristics of CPT from the nanoparticle formulations were investigated in a release medium with gradually changing $\mathrm{pH}$ representing the GIT corresponding to the actual transit times with dialysis membrane method at $37{ }^{\circ} \mathrm{C}$ in a shaking water bath (100 rpm) under sink conditions. Release experiments were performed by placing $3 \mathrm{~mL}$ CPTloaded CD nanoparticle formulation in the dialysis membrane. The closed bag was then immersed in release medium which provides external sink conditions with $\mathrm{pH} 1.2$ simulated gastric fluid (SGF) for a period of (0-2 h), followed by $\mathrm{pH} 4.5$ simulated intestinal fluid (SIF) for (2-4 h) and finally in $\mathrm{pH} 7.4$ simulated colonic fluid (SCoF) $(4-24 \mathrm{~h})$ at $37^{\circ} \mathrm{C}$ gradually to mimic physiological conditions that the nanoparticle will encounter once administered orally. At the end of each corresponding time point the dialysis membrane bag was transferred to the next release medium. $500 \mu \mathrm{L}$ of sample was taken from dialysis membrane at predetermined time points $(0.5,1,2,3,5,7,10$ and $24 \mathrm{~h})$ and replaced by equal volume of fresh release medium at the same temperature. The cumulative percentage of total CPT released for each time point was determined by HPLC simultaneously quantifying both the active lactone $\left(r^{2}=0.9989\right)$ and the inactive carboxylate $\left(r^{2}=0.9995\right)$ forms of the drug.

\section{Stability of CD nanoparticles in simulated gastrointestinal fluids}

Simulated gastrointestinal fluids (SGF, SIF, SCoF) were used to assess the influence of gastrointestinal fluids on the physical stability of $C D$ nanoparticles. $1 \mathrm{~mL}$ of each $C D$ nanoparticle formulation was added to $9 \mathrm{~mL}$ gastrointestinal medium and incubated for $2 \mathrm{~h}$ in case of SGF, $3 \mathrm{~h}$ in case of SIF and $8 \mathrm{~h}$ in case of SCoF. At the end of time points for each simulated medium, mean particle size $(\mathrm{nm}), \mathrm{PDI}$ and zeta potential $(\mathrm{mV})$ of the CD nanoparticles were measured and evaluated in reference with initial measurement before the incubation.

\section{Physical storage stability of CPT loaded CD nanoparticles}


In order to evaluate the short-term physical stability of the formulations, aqueous nanoparticle dispersion were stored at $4{ }^{\circ} \mathrm{C}$ for 30 days and the mean particle size (nm), PDI and zeta potential $(\mathrm{mV})$ were measured at predetermined intervals $(0,1,7,14$ and 30 days).

\section{Penetration of CD nanoparticles through artificial mucus layer}

Penetration of CPT loaded amphiphilic CD nanoparticles through an artificial mucus layer model was carried out as previously described (Ungaro et al., 2012). In this method artificial mucus layer was prepared and then placed on the gelatin layer. For this purpose, firstly, 10\% $(\mathrm{w} / \mathrm{v})$ gelatin dispersion was prepared by heating on magnetic stirrer in $50 \mathrm{~mL}$ of ultrapure water at $60^{\circ} \mathrm{C}$ and $1 \mathrm{~mL}$ dispersion was placed in each well of the 24-well cell plates. Gelatin dispersion was then cooled at room temperature to form the gelatin layer in the experimental model. Artificial mucus was prepared by dissolving $250 \mathrm{mg}$ of mucin, $0.295 \mathrm{mg}$ diethylenetriaminpentaacetic acid (DTPA), $250 \mathrm{mg} \mathrm{NaCl}, 110 \mathrm{mg} \mathrm{KCl}, 250 \mu \mathrm{L}$ sterile egg yolk emulsion and $1 \mathrm{~mL}$ RPMI in $50 \mathrm{~mL}$ of ultrapure water under magnetic stirring at room temperature. $1 \mathrm{~mL}$ artificial mucus was added to each well on the gelatin layer in the 24-well plates. The preparation of the artificial mucus layer model to which the experiment will be performed is completed by the addition of the artificial mucus solution onto the gelatin layer.

In order to examine the penetration of amphiphilic CD nanoparticles, $500 \mu \mathrm{L}$ of nanoparticle dispersion was added onto this artificial mucus model and left for incubation for $24 \mathrm{~h}$ at room temperature. After 24 hours, the artificial mucus containing nanoparticles was removed from each well. The lower gelatin layer was washed with ultrapure water $(2 \mathrm{~mL} \times 6$ replicate) to remove the mucus completely and then heated to $60{ }^{\circ} \mathrm{C}$ to make the solid gelatin again to liquefy. It was then centrifuged at $6000 \mathrm{rpm}$ for $20 \mathrm{~min}$ and the supernatant was removed. The amount of CPT both in lactone and in carboxylate form in the supernatant was analyzed by the validated HPLC method. Thus, by using the artificial mucus layer model experiment, the ability of nanoparticle dispersions, which is added to the mucus mixture on the gelatin layer to penetrate firstly through the mucus layer and then reach the gelatin layer, was evaluated by determination of the total CPT and stable fraction of the drug that can be transported into the gelatin layer.

\section{In vitro cell culture studies}


Cytotoxicity of blank CD nanoparticles was evaluated against L929 (American Type Culture Collection, USA) mouse fibroblast cell line. L929 cells were grown in Dulbecco's modified Eagle's medium (DMEM) containing $10 \%$ fetal bovine serum (FBS), penicillin (100 units $/ \mathrm{mL}$ ) and streptomycin $(100 \mu \mathrm{g} / \mathrm{mL})$ in $25 \mathrm{~cm}^{2}$ flasks. 5000 cells in $100 \mu \mathrm{L}$ DMEM were then seeded into each well of 96 -well plates. Then, the cells were incubated for 24 hours in a $5 \%$ $\mathrm{CO}_{2}$ incubator at $37^{\circ} \mathrm{C}$. At the end of 24 hours, cell culture medium in the wells was replaced with fresh medium containing blank CD nanoparticles at different dilution rates $(1: 16,1: 32$, 1:64 and 1:128). After 48 hours incubation, $25 \mu \mathrm{L}$ 3-(4,5-dimethylthiazol-2-yl)-2,5diphenyltetrazolium bromide (MTT) in PBS medium $(5 \mathrm{mg} / \mathrm{mL}$ ) was added to each well and incubated for 4 hours. Then, $200 \mu \mathrm{L}$ of DMSO was added to each well to dissolve the formazan crystals formed. Optical density (OD) was determined with the plate reader at 450 $\mathrm{nm}$. The cells incubated with DMEM were evaluated as the control. Cell viability (\%) was calculated according to the following equation;

Cell viability $(\%)=(O D$ of treated wells $/ O D$ of untreated cells $) \times 100$.

The anticancer activity of CPT-loaded and blank amphiphilic CD nanoparticles was researched with a cell culture study procedure aimed at comparing the activity of drug loaded nanoparticles with equivalent amounts of drug solution in DMSO at 2 different concentrations $(0.1 \mu \mathrm{g} / \mathrm{mL}, 0.5 \mu \mathrm{g} / \mathrm{mL})$ and incubation for $48 \mathrm{~h}$ on the HT-29 human colon cancer cell line. HT-29 cells were seeded in 96 -well plates at $10^{4}$ cells $/ 100 \mu \mathrm{L}$ per well and incubated for 24 hours to attach the cells in the wells.

CPT-loaded CD nanoparticle formulations are diluted with DMEM containing $10 \%$ BSA in four different concentrations to achieve equivalent concentrations $0.1 \mu \mathrm{g} / \mathrm{mL}$ and $0.5 \mu \mathrm{g} / \mathrm{mL}$ camptothecin $100 \mu \mathrm{L}$ for either CPT solution or nanoparticle (blank and drug loaded). The cells were incubated with CPT-loaded and blank nanoparticle formulations and CPT solutions for $48 \mathrm{~h}$.

At determined time points, $10 \mu \mathrm{L}$ of water soluble tetrazolium salt (WST-1) was added to each well and allowed to incubate for $4 \mathrm{~h}$. Then optical density (OD) at $450 \mathrm{~nm}$ was determined by the cell plate reader (Molecular Devices, USA). Untreated cells, incubated with only cell medium, were accepted as control with $100 \%$ cell viability. Cell viability (\%) was calculated according to the following equation; 
Cell viability $(\%)=(O D$ of treated wells $/ O D$ of untreated cells $) \times 100$.

\section{Evaluation of in vitro transport of CPT across Caco-2 cell line}

In vitro transport evaluation of amphiphilic CD nanoparticles was carried out across the human adenocarcinoma Caco-2 cell line. Caco-2 cells, which are passage number 5, were passaged and counted. Cells were seeded into 12-well polycarbonate membrane filters (Thincerts ${ }^{\mathrm{T}}$, pore diameter $1 \mu \mathrm{m}$, area $1.13 \mathrm{~cm}^{2}$ ) for permeability studies at a density of 60.000 cells/insert. $500 \mu \mathrm{L}$ of culture medium for apical part of the wells and $1 \mathrm{~mL}$ of culture medium for basolateral part were placed into the inserts. Mediums both in the apical and basolateral sections were changed every 48 hours for 18-21 days and incubated in an incubator at $37{ }^{\circ} \mathrm{C}$ and $5 \% \mathrm{CO}_{2}$. When a monolayer of Caco-2 cell formed, cell integrity was assessed through transepithelial electrical resistance (TEER) measurement before the transmembrane permeability experiments. TEER values were measured by EVOM ${ }^{2}$ Epithelial Voltohmmeter (WPI World Precision Instruments, USA). Permeability experiments were initiated when TEER values reached at least $300 \Omega \cdot \mathrm{cm} 2$ on the 18th day of the cell seeding.

Both CPT solution and CPT loaded CD nanoparticle formulations were diluted to contain 20 $\mu \mathrm{g} / \mathrm{mL}$ CPT. Hank's balanced salt solution (HBSS) was placed on the apical and basolateral compartments and left for about 30 minutes. Subsequently, CPT solution and CPT loaded CD nanoparticle formulations were added to the apical region prepared in $0.5 \mathrm{~mL}$ HBSS, and only $1 \mathrm{~mL}$ HBSS was added to the basolateral region.

Cells were incubated at $37{ }^{\circ} \mathrm{C}$ for 4 hours. Samples were taken from the basolateral section at the end of the incubation and analyzed by HPLC. All experimental groups were studied in triplicate. The apparent permeability coefficients (Papp, $\mathrm{cm} / \mathrm{s}$ ) for each experimental group were calculated as according to the following equation;

$$
\text { Papp }=d Q / d t .1 /\left(A . C_{0}\right)
$$

Where $\mathrm{dQ} / \mathrm{dt}$ is the permeability rate, $\mathrm{Co}$ is the initial concentration on the apical side and $\mathrm{A}$ is the surface area of the monolayer.

\section{Results and Discussion}

In vitro characterization of CPT loaded amphiphilic CD nanoparticle 
Considering the characterization parameters of CPT-loaded nanoparticles; smallest particle size $(134.6 \pm 19.3 \mathrm{~nm})$ and PDI $(0.27 \pm 0.03)$ with the highest positive zeta potential value $(+40.2 \pm 0.32 \mathrm{mV})$ was obtained for Poly- $\beta-C D-C 6$ nanoparticles. In previous studies with similar amphiphilic cyclodextrins, the smallest particle size could be obtained with the Polyß-CD-C6 in a similar manner (Varan et al., 2017a).

Hydrophilic-lipophilic balance (HLB) of the amphiphilic cyclodextrins was previously reported to be crucial on the characteristic parameters of the nanoparticles obtained with amphiphilic cyclodextrins whose primary face or secondary faces were modified (Memişoğlu et al., 2002; Pflueger et al., 2016). Similarly, in our study, HLB values are variable due to the differences in the molecular structure of amphiphilic cyclodextrins. HLB was defined by Griffin as the equilibrium between the hydrophilic and lipophilic portions of amphiphilic molecules and is calculated according to the following equation (Griffin, 1949, 1954).

$\mathrm{HLB}=20 \times \mathrm{M}_{\mathrm{h}} / \mathrm{M}$

According to this equation; $M_{h}$ is the molecular mass of the hydrophilic portion and $M$ is the molecular mass of the whole molecule, giving a result on a scale of 0 to 20 . When considered in this point of view, the HLB for 6-O-CAPRO- $\beta-C D$, whose only primary face was modified with 7 hexanoyl groups, was 10.1, and for the Poly- $\beta-C D-C 6$, both the primary face of which was modified with 7 cationic amine groups and the secondary side with 14 hexanoyl groups, the HLB value was 15.8. In this context, when using Poly- $\beta-C D-C 6$, which has a larger HLB, it is thought that interfacial tension between the surfaces where nanoparticle formation occurs is reduced more effectively than single face modified derivative 6-O-CAPRO- $\beta-C D$. As a result, it was thought that smaller particles were obtained with a narrower monodispersity by nanoprecipitation process.

In the case of PEl coated nanoparticles, dispersions having a larger particle size, a wide particle size distribution with a high polydispersity index were obtained. This is based on the suggestion that the viscosity of the aqueous phase during preparation increases as a result of the addition of viscous PEI dispersion, thus resulting in larger nanoaggregates obtained through the nanoprecipitation method. When this finding is considered together with the information available in the literature on the nanoprecipitation method, the increase in the viscosity of the aqueous phase is thought to have a negative effect on the solvent diffusion, 
which allows the formation of nanoparticles (Salatin et al., 2017). Therefore, it has been evaluated that the nanoparticles show a high polydispersity with large particles due to the inability to obtain them in a stable nanoprecipitation conditions. As a result, 6-O-CAPRO- $\beta$ CD nanoparticles could be coated with PEI, however intended parameters could not be achieved (Salatin et al., 2017; Tan et al., 2016).

Table 1. Mean particle size, PDI and zeta potential of blank and CPT-loaded CD nanoparticles prepared with optimum parameters determined after pre-formulation studies (Organic solvent is acetone, CD concentration is $0.1 \%(w / v)$, CS concentration is $0.025 \%(w / v)$, PEI concentration is $2.5 \%(w / v))(n=3, \pm S D)$.

\begin{tabular}{|c|c|c|c|c|}
\hline \multicolumn{2}{|c|}{ Nanoparticle Formulations } & \multirow{2}{*}{\begin{tabular}{|c|}
$\begin{array}{c}\text { Particle } \\
\text { Diameter } \pm \text { SD } \\
(\mathrm{nm})\end{array}$ \\
$194 \pm 3$
\end{tabular}} & \multirow{2}{*}{$\begin{array}{c}\text { PDI } \pm \text { SD } \\
0,11\end{array}$} & \multirow{2}{*}{$\begin{array}{c}\mathrm{ZP} \pm \mathrm{SD}(\mathrm{mV}) \\
\frac{-24 \pm 2}{}\end{array}$} \\
\hline & Blank & & & \\
\hline & CPT loaded & $252 \pm 12$ & 0,27 & $-22 \pm 2$ \\
\hline \multirow{2}{*}{ CS-(6-O-CAPRO- $\beta-C D)$} & Blank & $284 \pm 13$ & 0,23 & $+18 \pm 1$ \\
\hline & CPT loaded & $298 \pm 23$ & 0,29 & $+19 \pm 1$ \\
\hline \multirow{2}{*}{ PEI-(6-O-CAPRO- $\beta$-CD) } & Blank & $205 \pm 6$ & 0,30 & $+22 \pm 1$ \\
\hline & CPT loaded & $273 \pm 42$ & 0,39 & $+22 \pm 1$ \\
\hline \multirow{2}{*}{ Poly- $\beta-C D-C 6$} & Blank & $117 \pm 9$ & 0,34 & $+42 \pm 3$ \\
\hline & CPT loaded & $135 \pm 19$ & 0,27 & $+40 \pm 1$ \\
\hline
\end{tabular}

\section{Determination of drug loading capacity}

Encapsulation efficiency (EE), which is one of the crucial characterization parameters for drug delivery systems, is the basic parameter for calculating the amount of drug encapsulated into nanoparticle for in vitro and in vivo experiments and dose calculations. Encapsulation efficiency should be determined reliably in order to evaluate the efficacy of the method and to determine the amount of drug carried by the nanoparticles for eventual in vivo and clinical studies (Zhang and Feng, 2006).

Chemical structure of the amphiphilic CD derivative, nanoparticle preparation method, affinity of the active substance, hydrophilic/lipophilic balance and physicochemical 
properties of the active substance are known to influence on the efficiency of encapsulation in nanoparticle preparation with amphiphilic $\beta-C D$ derivatives (Memişoğlu et al., 2003). Encapsulation efficiencies of nanoparticle formulations are presented in Table 2. The two formulations with the highest CPT encapsulation efficiency were CS-6-O-CAPRO- $\beta$-CD (36\%) and Poly- $\beta$-CD-C6 (35\%). PEI-6-O-CAPRO- $\beta$-CD nanoparticles were found to have a lower encapsulation efficiency compared to other formulations because of the ineffectiveness of the nanoprecipitation method with PEI $(p<0,05)$. In the CS-6-O-CAPRO- $\beta$-CD nanoparticles, which have the highest encapsulation efficiency, this situtation was interpreted as a second drug layer adsorbed onto the surface with the effect of chitosan polymer used as coating material so the drug was either encapsulated and/or adsorbed onto nanoparticles. Although no coating material was used, relatively high encapsulation efficiency of CPT was achieved for Poly- $\beta-C D-C 6$ nanoparticles.

The difference between the encapsulation efficiency of the coated 6-O-CAPRO- $\beta-C D$ and uncoated Poly- $\beta-C D-C 6$ nanoparticles is thought to result from differences in the molecular structure of both amphiphilic CDs. 6-O-CAPRO- $\beta$-CD was modified at the primary face with 7 lipophilic groups, while Poly- $\beta-C D-C 6$ was modified with 7 cationic groups on the primary surface and 14 lipophilic groups on the secondary surface (Varan et al., 2017b). Poly- $\beta-C D-C 6$ has more hydrophobic chains because it carries twice the number of aliphatic chains than 6O-CAPRO- $\beta$-CD. Achieving higher encapsulation efficiency with Poly- $\beta-C D-C 6$ compared to 6O-CAPRO- $\beta-C D$ is explained by its stronger interaction with hydrophobic CPT.

Considering from another side, it should be mentioned that Poly- $\beta-C D-C 6$ show also more hydrophilic characteristic since the amine-coated primary face is heavily cationic and this face can interact with CPT strongly by electrostatic interactions in terms of encapsulation process. As a result of comparison, it can be said that the amphiphilic characteristic of Poly$\beta-C D-C 6$ is heavier than that of $6-O-C A P R O-\beta-C D$, and this situation is thought to be very decisive in terms of encapsulation efficiency and cause different results among nanoparticle formulations. 
Table 2. Encapsulation efficiency (\%) of amphiphilic CD nanoparticles for CPT (CD amount is $1 \mathrm{mg} / \mathrm{mL}$ and initial CPT amount is $0.1 \mathrm{mg} / \mathrm{mL}$ in all formulations) $(n=3, \pm S D)$.

\begin{tabular}{|c|c|}
\hline Nanoparticle Formulations & Encapsulation efficiency \% $\mathbf{\text { SD }}$ \\
\hline 6-O-CAPRO- $\beta$-CD & $32,13 \pm 2,8$ \\
\hline CS-(6-O-CAPRO- $\beta-C D)$ & $36,56 \pm 2,4$ \\
\hline PEI-(6-O-CAPRO- $\beta-C D)$ & $21,04 \pm 3,3$ \\
\hline Poly- $\beta-C D-C 6$ & $35,11 \pm 3,2$ \\
\hline
\end{tabular}

\section{In vitro release studies of CPT from amphiphilic CD nanoparticles}

In vitro release studies were carried out for 24 hours. In order to simulate the GIT conditions in terms of both $\mathrm{pH}$ and transition time, in vitro release study was performed for 0-2 hours in SGF, 2-5 hours in SIF and then under SCoF conditions until the end of the experiment. The aim of the release study is to reveal whether the formulation, are able to preserve the drug encapsulated in the stomach and small intestine, and preferentially release it when it reaches the colon. Optimum formulation was expected to carry a majority of CPT active lactone form to the colon.

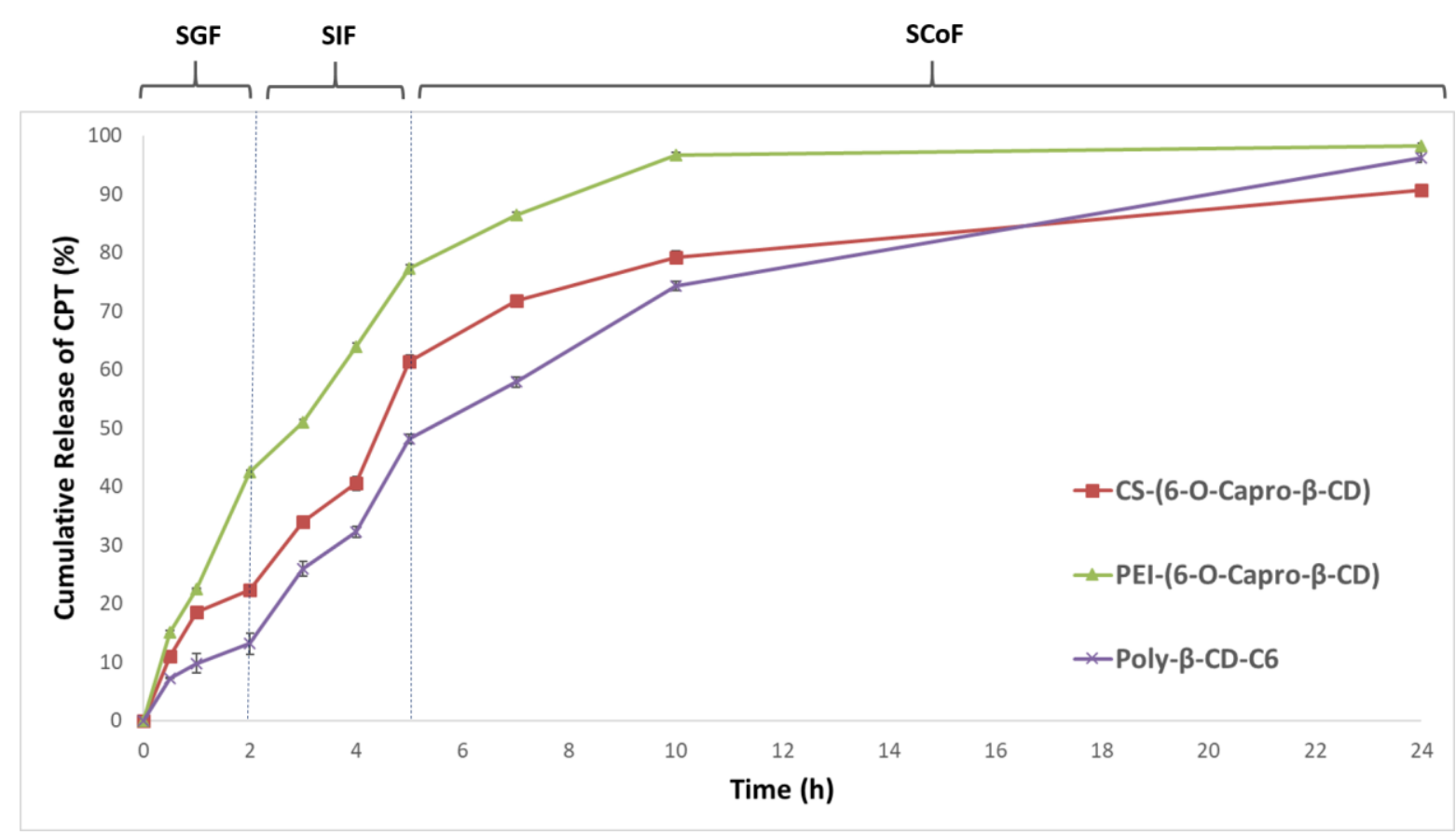

Figure 2. Cumulative release profile of CPT from different amphiphilic CD nanoparticles $(n=3, \pm S D)$. 
The majority of the drug release occurred in the first 5 hours particularly in SIF for all formulations. The slowest release profile belonged to the Poly- $\beta-C D-C 6$ nanoparticles. The cumulative amount of CPT released in SGF and SIF was found as $61 \%$ for CS-(6-O-CAPRO- $\beta$ CD), 77\% for PEI-(6-O-CAPRO- $\beta-C D)$ and 48 for Poly- $\beta-C D-C 6$ as seen in Figure 2.

It is known that oral formulations should take approximately 5 hours to reach the colon, and that the first 2 hours of this time are spent in the stomach and 3 hours in the small intestine (Belali et al., 2019). Additionally, drug delivery systems are exposed to a broad $\mathrm{pH}$ range that tends to increase from the stomach towards the colon (stomach pH 1.2, small intestine $\mathrm{pH}$ 4.5 , column pH 7.4) (Fallingborg, 1999). At the end of the $5^{\text {th }}$ hour PEI-6-O-CAPRO- $\beta-C D$ and CS-6-O-CAPRO- $\beta$-CD nanoparticles showed faster release profiles $(p>0.05)$ than Poly- $\beta-C D-C 6$ nanoparticles. This faster release profile can be attributed to the burst release of the CPT, which is adsorbed to the coating material surface. Additionally, PEI-6-O-CAPRO- $\beta$-CD nanoparticles released $77 \%$ of the CPT before it reached the colon and did not show a favorable release profile. Poly- $\beta-C D-C 6$ nanoparticles released a smaller amount of CPT $(48 \%)$ before the colon medium as compared to the other formulations $(p<0.05)$. As Poly- $\beta-$ CD-C6 formulation can deliver the maximum amount of stable CPT to the colon, it was considered as the optimal formulation.

In the literature, there are studies reporting that the release slows down with the increase of the particle size of the nanoparticles, and the release speeds up due to the increase of the specific surface area with the decrease of the particle size (Mora-Huertas et al., 2010; Varan et al., 2017a). However, in this study, cationic Poly- $\beta$-CD-C6 with the smallest particle size has a slower release profile than the other formulations. This situation is believed to be related to other parameters that affect release profiles except particle size. The primary and secondary faces of Poly- $\beta-C D-C 6$ have been modified with aliphatic chains and it has been associated with the slowest release despite the small particle size. In this context, the fact that the drug molecule showed high affinity for cyclodextrin as a result of the interaction of CPT with both the CD cavity and the modifying groups was interpreted as the cause of the slow release profile. Varan et al. reported on comparative drug release profiles of another lipophilic anticancer drug paclitaxel from Poly- $\beta-C D-C 6$ amphiphilic CD nanoparticles and non-ionic amphiphilic CD nanoparticles with negative surface charge (Varan et al., 2017a). At the end of the study, it has been stated that the nanoparticle with smaller particle size 
shows slower paclitaxel release due to the stronger affinity and electrostatic interaction between the drug and the cationic $C D$, supporting our interpretation.

\section{Stability of CD nanoparticles in simulated gastrointestinal fluids}

One of the primary conditions for development of an effective system for oral drug administration is the stability of both the drug carrier system and the encapsulated drug during storage and administration. Therefore, nanoparticulate drug delivery systems intended to be administered orally should be able to maintain physical stability under various conditions of the gastrointestinal tract (Plapied et al., 2011). As explained previously, the most important barriers to oral drug administration are the complex physiological structure of the gastrointestinal tract and the physiological barriers such as $\mathrm{pH}$, enzymes, mucin, salts, and lipids. Critical quality attributes of nanoparticles such as particle size, PDI and zeta potential, which were exposed to the GIT mimicking conditions, are given in Figure 3.

Characterization parameters of the nanoparticles according to the results of physical stability tests in gastrointestinal simulated fluids; PEI-6-O-CAPRO- $\beta-C D$ nanoparticles showed significant changes in SIF and SCoF $(p<0.05)$. Significant change in physicochemical characters of the CS-6-O-CAPRO- $\beta-C D$ nanoparticles was observed only in simulated colonic fluid $(p<0.05)$, whereas Poly- $\beta-C D-C 6$ remained almost unchanged in simulated gastrointestinal fluids.

The mean particle size and PDI of all the formulations displayed a tendency to increase due to the challenging environmental conditions ( $\mathrm{pH}$ change, ions, salt effect etc.) and incubation time of simulated gastrointestinal fluids. Additionally nanoparticles other than Poly- $\beta-C D-C 6$ formulation may have a tendency to aggregate due to their zeta potential below $\pm 30 \mathrm{mV}$ (Honary and Zahir, 2013). It was observed that zeta potentials tend to decrease for all formulations treated with simulated fluids compared to initial values. However, zeta potential value of the Poly- $\beta-C D-C 6$ formulation $+44.2 \mathrm{mV}$ initially and $+39.9 \mathrm{mV}$ in the simulated colon fluid at the end of 8 hours was still sufficiently high for a stable colloidal system. Similarly, Lazzari et al. evaluated the stability of polymeric nanoparticles in gastrointestinal fluids (Lazzari et al., 2012). There was $20 \%$ increase in the particle size of the poly-lactic acid (PLA) (-18 mV) nanoparticles due to aggregation after 24 hours incubation, 
but there was no statistical increase in the poly-methylmethacrylate (PMMA) (-38 mV) nanoparticles. This has been reported to arise from the higher zeta potentials of PMMA nanoparticles compared to PLA nanoparticles.

Poly- $\beta-C D-C 6$ nanoparticles can be a physically stable drug delivery system while maintaining stability in all simulated gastrointestinal fluids in terms of mean particle size, polydispersity index and zeta potential values. Therefore, it was considered that the drug delivery system could have a positive effect on the physiological efficacy of camptothecin due to the residence time and chemical stability of camptothecin, which can remain, in the encapsulated state for a longer period without deteriorating its chemical structure in the gastrointestinal tract.
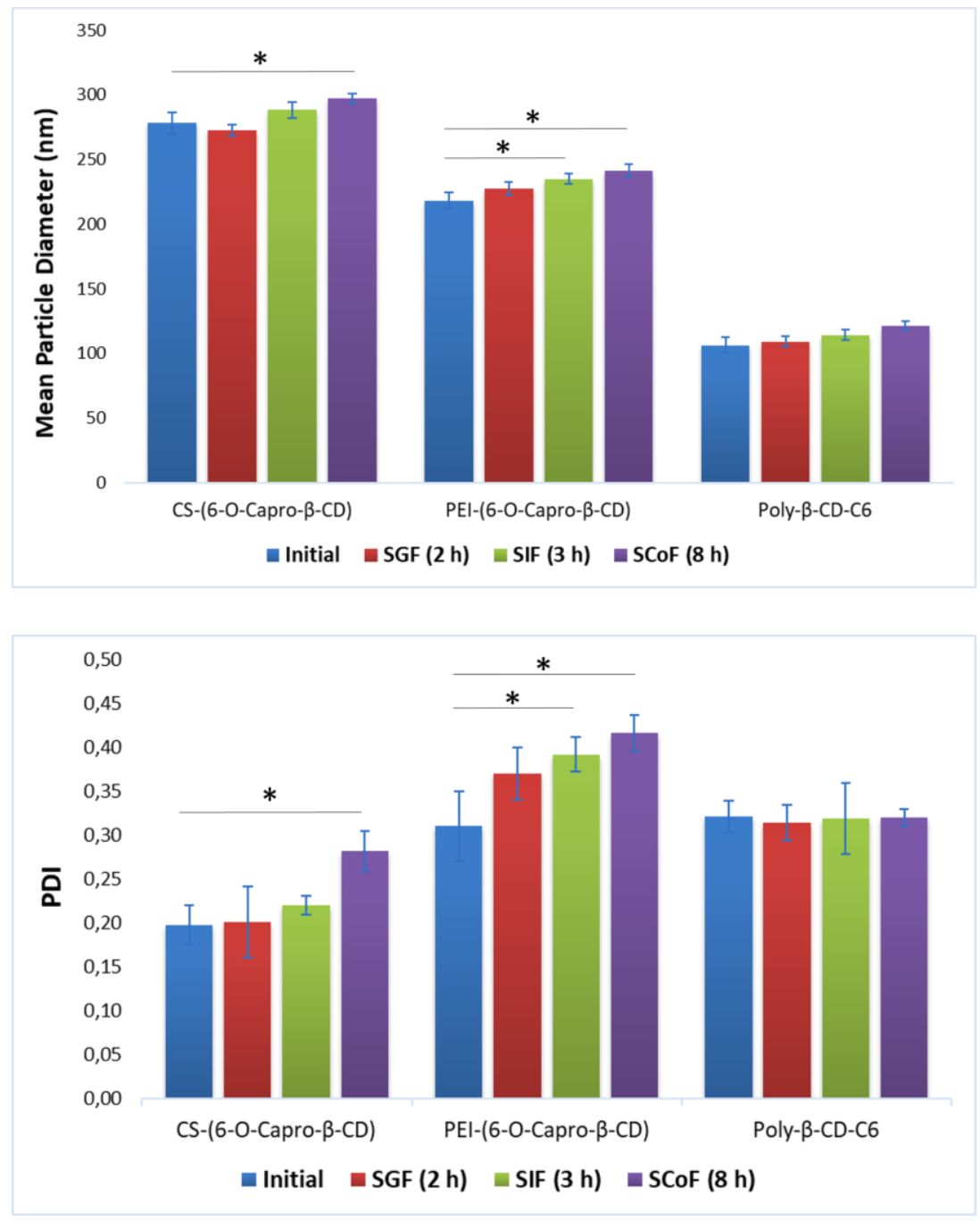


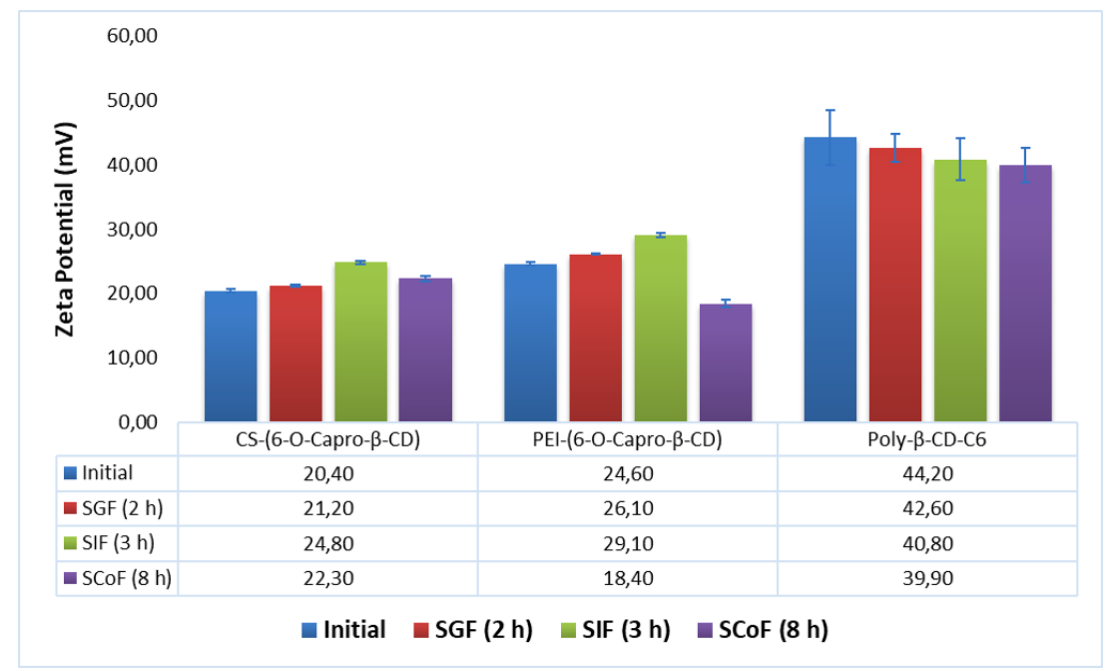

Figure 3. Particle size, PDI and zeta potential values of the different formulations, which were, exposed to the GIT conditions ( $n=3, \pm S D)(* ; p<0.05)$.

\section{Physical stability of CPT loaded CD nanoparticles}

Short-term stability results performed for 30 days are given in Figure 4 . There was no significant change in CS-6-O-CAPRO- $\beta$-CD and Poly- $\beta-C D-C 6$ nanoparticles on days 1,7 and 14. On the other hand, a significant change $(p<0.05)$ was determined at day 30 for CS-6-OCAPRO- $\beta-C D$ and Poly- $\beta-C D-C 6$. For the PEI-6-O-CAPRO- $\beta-C D$ nanoparticles, although no statistically significant change, mean particle size, PDI and zeta potential remained significantly higher than the other formulations. Additionally, the PEI-6-O-CAPRO- $\beta-C D$ nanoparticles had a polydispersity index of 0.41 by the end of 30 days, due to the low zeta potential $(+13.4 \mathrm{mV})$, the aggregation of the particles over time and the particle size distribution spread over a wide range.

It was observed that the mean particle size was $149.8 \mathrm{~nm}$ and polydispersity index was 0.274 at the end of 30 days for Poly- $\beta-C D-C 6$ nanoparticles and it could maintain lower particle size and higher zeta potential values more effectively than the other formulations. Poly- $\beta-C D-C 6$ nanoparticles had an initial zeta potential value of $+40.2 \mathrm{mV}$ and was measured as $+37.9 \mathrm{mV}$ on the $30^{\text {th }}$ day, which is higher than the other two formulations. Thus Poly- $\beta-C D-C 6$ can be recommended as the physically stable formulation among the all CD nanoparticles probably due to its higher net surface charge and small particle size by the side of initial values. 



Figure 4. Particle size, PDI and zeta potential values of the different formulations for 30 days $(n=3, \pm S D)(*$; $p<0.05)$.

\section{In vitro evaluation of nanoparticle interaction with mucus}

For oral formulations, it is important to remain stable and prolong the stay in the GIT. Thus, the duration of interaction of the nanoparticulate system with gastrointestinal epithelial 
cells and biological membranes can be increased and sufficient time for effective release of the encapsulated drug in the delivery system can be provided. One of the most important obstacles to the effectiveness of many orally administered drug delivery systems is that they are rapidly eliminated physically from the system as a result of gastrointestinal motility (Luo et al., 2014). The interaction of nanoparticles with mucin in the gastrointestinal tract may prolong the residence time of the drug delivery system in the region. It should then be able to penetrate the mucus layer and reach the epithelial cell layer. It is known that nanoparticulate drug delivery systems can alter their interaction with mucin by surface modifications and/or alteration of surface charge and the mucoadhesive characteristics of drug delivery systems can be modified.

In the literature, it is stated that nanoparticulate drug delivery systems with positivelycharged surface or systems coated with a positively charged polymer interact with mucin and mucus layer at a higher rate and as a result of this they can stay in the intestinal lumen for a longer time (Borges et al., 2005). Unal et al. showed that the positively-charged nanoparticle formulations increased interaction with mucin (Ünal et al., 2015a). Similarly, in another study conducted by Luo et al. for oral chemotherapy, it was reported that mucin was negatively charged (approximately $-50 \mathrm{mV}$ ) due to the sulfhydryl groups contained in it, and interaction with mucin could be increased by the cationic particles for the purpose of bioadhesion in the preparation of oral formulations (Luo et al., 2014).

Orally administered nanoparticles are expected to reach the intestinal epithelial cell layer after prolonged stay in the intestinal lumen region by interacting with mucin sufficiently. If the interaction with mucin is too tight, it becomes difficult for the nanoparticles trapped in the mucin to penetrate the mucus layer and then reach the intestinal cell layer. The steps of sufficient interaction with the mucin described above and subsequent penetration into the mucus layer are two interrelated and sequential processes that should be in an equilibrium state (Ungaro et al., 2012).

According to the study performed in order to evaluate penetration of nanoparticles to mucus layer; the highest penetration rate was found to be Poly- $\beta-C D-C 6$ formulation with a penetration rate of $73 \%$. The CS-6-O-CAPRO- $\beta-C D$ formulation was the second highest penetrating formulation with a penetration rate of $65 \%$ and PEI-6-O-CAPRO- $\beta-C D$ formulations penetrated the artificial mucus layer by $45 \%$. The strong cationic surface charge 
of the Poly- $\beta-C D-C 6$ and CS-6-O-CAPRO- $\beta-C D$ formulations led a strong electrical interaction with the mucus resulting in the high penetration of the drug.

In order to evaluate the penetration of the nanoparticles to the artificial mucus layer, the amount of lactone and carboxylate forms of CPT was calculated separately by the validated HPLC method to elucidate the amount of the active and inactive forms of the drug which is available in the receptor compartment. In terms of proportion of active lactone form, the formulation with the highest total penetration rate was observed for Poly- $\beta-C D-C 6$ as $73 \%$, of which $52 \%$ was quantified as the active form.

Penetration enhancing activities of CDs were demonstrated by literature studies and the results obtained at the end of the experiment can be supported (Loftsson and Brewster, 2011). In a study investigating the intestinal permeability increasing effect of CDs (Mazzaferro et al., 2012), it was reported that the intestinal permeability of the docetaxel increased significantly with the CD nanoparticles in the rat intestinal ex vivo model. Similarly, in the study conducted by Unal et al. mucosal penetrations of Polycaprolactone (PCL), 6-OCAPRO- $\beta$-CD and CS-6-O-CAPRO- $\beta$-CD nanoparticle formulations were compared. At the end of the study performed in the artificial mucus model, the highest mucosal penetration has been obtained in CS-6-O-CAPRO- $\beta-C D, 6-0-C A P R O-\beta-C D, P C L$ nanoparticle formulations, respectively. This showed that the intestinal permeability can be increased with $C D$, and that the interaction of nanoparticles with mucin, which gained a positive surface charge with CS coating, will increase and enables higher mucosal penetration (Ünal et al., 2015a).

In addition to the surface charge and cyclodextrin effect, the small particle size of the nanoparticles is thought to be effective in interaction with mucin and penetration into the mucus layer (Bandi et al., 2020). It has been assessed that the high penetration ability of the Poly- $\beta$-CD-C6 nanoparticle formulation prepared directly from amphiphilic cyclodextrin without any coating material having the smallest particle size and highest positive zeta potential may result from the described properties.

In order to develop an orally administered nanoparticulate system, the mucoadhesive feature of the nanoparticles and penetration into the mucus is a process that must be sequential and balanced with each other. For this reason, it is known that the nanoparticles 
has a mucoadhesive feature in the intestinal lumen and that the interaction with the mucin is at a sufficient level and then the penetration of the mucus should occur (Lai et al., 2009).

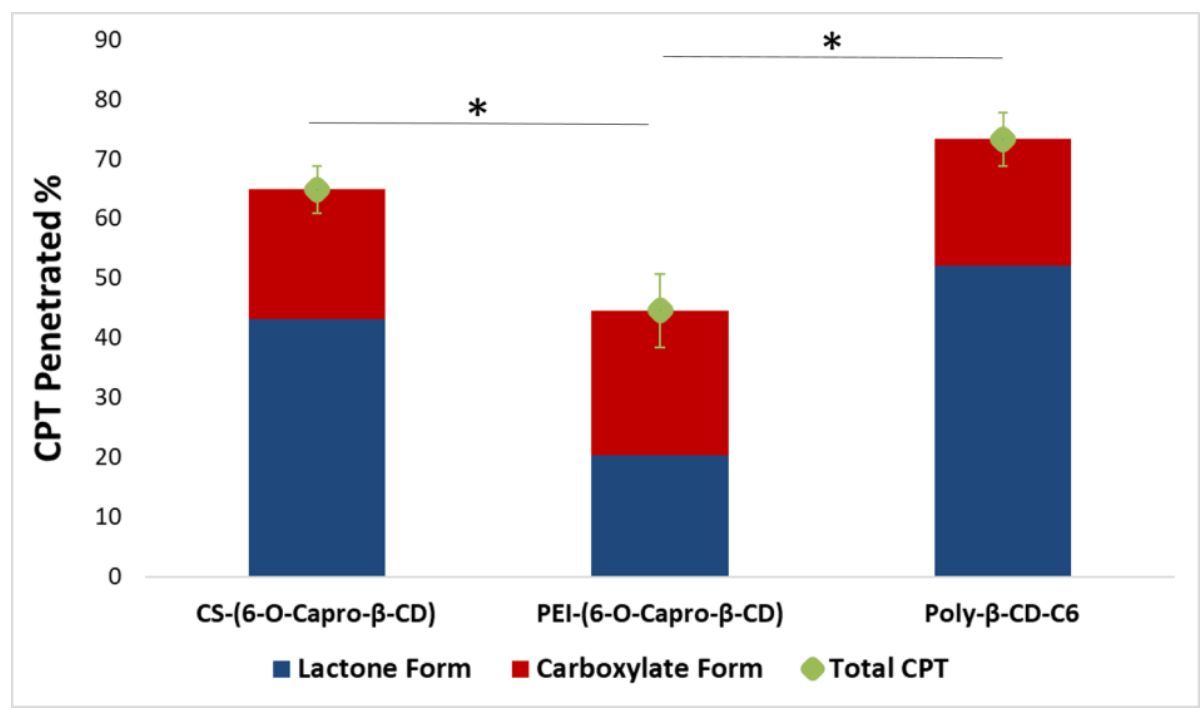

Figure 5. Amount of CPT penetrated through a artificial mucus gelatin layer from different formulations ( $n=3$, $\pm \mathrm{SD})(* ; p<0.05)$.

\section{In vitro cell culture studies}

\section{Cytotoxicity of blank CD nanoparticles against L929 cell line}

Cytotoxicity of blank CD nanoparticles was assessed on the 2929 cell line to predict their safety before the in vivo studies as recommended by USP (28). Safety studies on the L929 cell line are used as the standard method to evaluate the safety and cytotoxic effects on healthy cells of nanoparticulate systems designed to be applied to humans. In the literature, $80 \%$ cell viability is considered critical limit and the nanoparticle system is considered safe in above this treshold (Reul et al., 2011).

As seen in Figure 6.; Poly- $\beta-C D-C 6$ nanoparticles caused significant $(p<0.05)$ cell death compared to the control only at a 1:16 dilution ratio. Lower concentrations of Poly- $\beta-C D-C 6$ nanoparticles were found to be safe as they didn't cause significant cell death in reference to the control $(p>0.05)$ and the cell viability was still over $83 \%$. On the other hand, PEI-6-OCAPRO- $\beta$-CD nanoparticles reduced to range of $48 \%-59 \%$ at all dilutions. On the 2929 cell line recommended by American Pharmacopoeia (USP) 28 for the purpose of evaluating the 
safety of polymeric materials that will contact the patient directly or indirectly; CS-6-OCAPRO- $\beta-C D$ nanoparticles at a dilution of $1: 16$, Poly- $\beta-C D-C 6$ nanoparticles at a dilution of 1:32 were considered as safe, and the cell viability was over $80 \%$ (Reul et al., 2011).

According to the findings presented in Figure 6., PEI-6-O-CAPRO- $\beta-C D$ nanoparticles showed a significant cytotoxic effect compared to the control group and other nanoparticle formulations applied at the same dilution rate in all dilution rates. In the studies carried out in the literature about PEI used as surface coating polymer to give cationic properties to nanoparticles prepared, it is mentioned that the toxic effects of PEI can be observed and studies for the development of new and safe PEI derivatives are going on (Okon et al., 2014). On the contrary, the lack of such toxicity in CS coated 6-O-CAPRO- $\beta$-CD nanoparticles reveals the cytotoxic effect of the PEI. For this reason, PEI, which we use as cationic coating polymer, has been evaluated as the cause of serious cytotoxicity, which is seen as a result of direct and high rates of effect of nanoparticles on cells.

CS-6-O-CAPRO- $\beta$-CD and Poly- $\beta$-CD-C6 nanoparticles do not cause a severe cell death to be considered as toxicity at 1:32 dilution and lower concentrations. It was determined that the cell viability was $83 \%, 110 \%, 85 \%$ at $1: 32$ dilution and the cell viability tend to be higher at lower concentrations of CS-6-O-CAPRO- $\beta-C D$ and Poly- $\beta-C D-C 6$ nanoparticles. Interestingly the viability of the cells incubated with CS-6-O-CAPRO- $\beta-C D$ was higher than the control cells and this is thought to be the due to the nutritional property and positive effects of chitosan for the cells (Gao et al., 2012).

As a result of evaluating the safety of blank nanoparticle formulations applied at different dilution ratios for 24 hours on $\mathbf{L} 929$ cell line, two formulations other than PEI-6-O-CAPRO- $\beta$ $\mathrm{CD}$ nanoparticles can be used as safe drug delivery systems. It has been interpreted that the cytotoxic effect decreased inversely proportional to the dilution ratios the CS-6-O-CAPRO- $\beta$ $C D$ or Poly- $\beta-C D-C 6$ nanoparticle suspensions are expected to be further diluted in the body fluids and the toxicity profile will therefore be reduced and thus it is considered as safe drug delivery systems (Bilensoy et al., 2008). Considering the dilution rates of blank nanoparticles evaluated on healthy 2929 cells, CPT concentrations that they can carry at an optimal dilution rate of $1: 32$ were calculated as follows; CS-6-O-CAPRO- $\beta-C D(0.56 \mathrm{mcg} / \mathrm{mL})$, PEI-6-OCAPRO- $\beta$-CD $(0.33 \mathrm{mcg} / \mathrm{mL})$, Poly- $\beta-C D-C 6(0.55 \mathrm{mcg} / \mathrm{mL})$ for the further antiproliferative activity studies. 


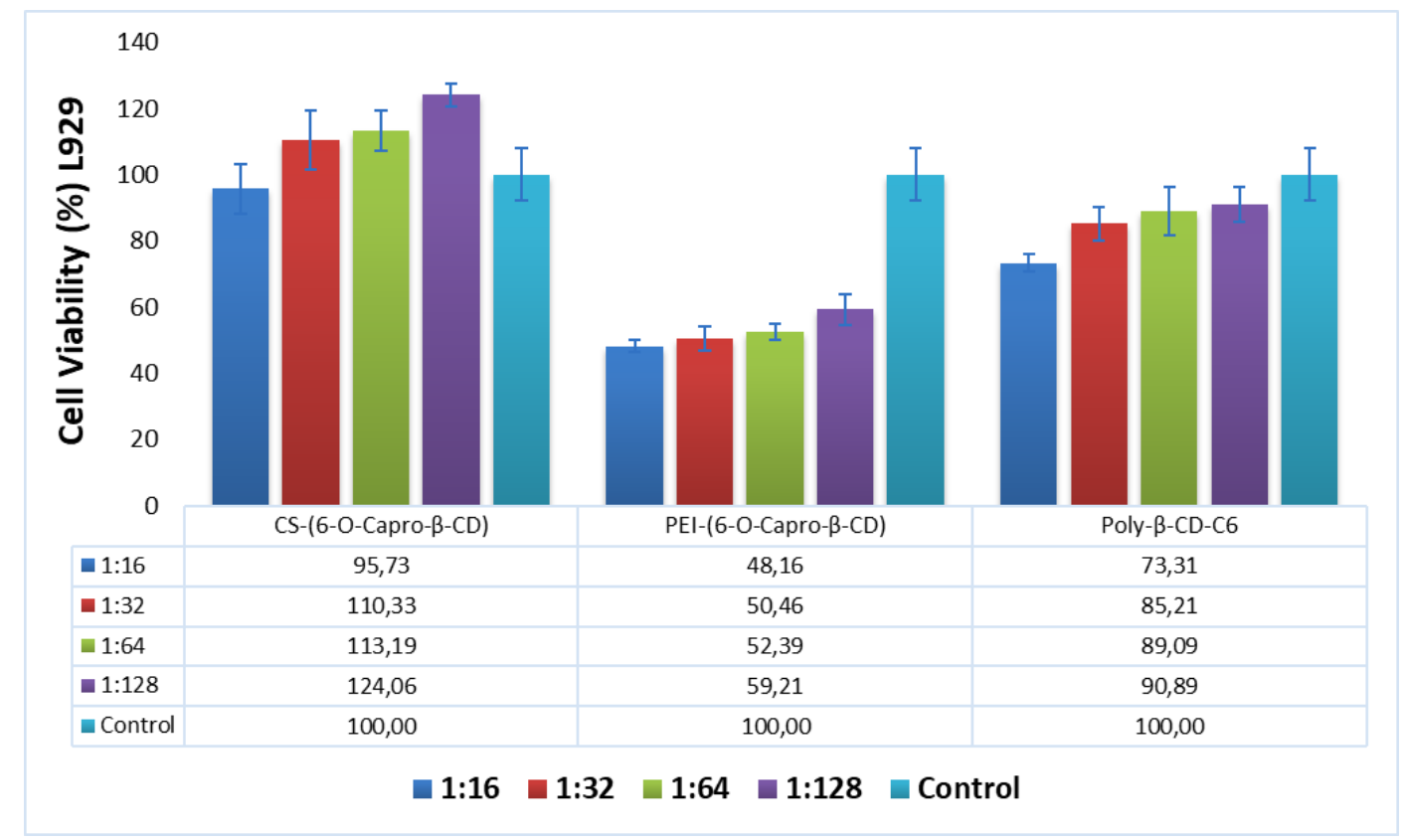

Figure 6. Cytotoxicity of blank amphiphilic CD nanoparticles on L929 cell line with MTT assay $(n=3, \pm$ SD).

\section{Antiproliferative Effect of CPT loaded CD Nanoparticles against HT-29 cell line}

In order to evaluate the antiproliferative activity of the CPT-loaded and blank nanoparticles, efficacy studies were performed in comparison with the equivalent concentration of camptothecin solution on the HT-29 human colon cancer cell line. CPT-loaded and blank nanoparticle formulations were compared with the equivalent concentration of CPT solution for $48 \mathrm{~h}$ incubation time and the results are presented in Figure 7.

In the evaluation of the antiproliferative activities of the nanoparticle formulations, a 48 hours incubation time was determined by taking into account the 'doubling time' period and the in vitro release profiles of the nanoparticles. In the literature, 'doubling time' is stated as 2-4 days for HT-29 cells (Forgue-Lafitte et al., 1989). This time refers to the proliferation time during which the number of cells can double and is important for the significance of the results in evaluating the antiproliferative activities of the formulations.

When considered in terms of in vitro release profiles, amphiphilic CD nanoparticle formulations provided a sustained release profile for 24 hours, completing the release of CPT 
from nanoparticles and evaluating its activity by accumulating in the cell with incubation period in the evaluation of anticancer efficacy. It is known that CPT, as a topoisomerase I inhibitor, shows cytotoxic effect with S-phase inhibitor activity in the cell cycle where DNA synthesis takes place (Minelli et al., 2012). Lalloo et al. also indicated that the S-phase, where CPT can exhibit anticancer activity by inhibition of DNA synthesis, is a short-term phase in the cell cycle, so that the cell death can be increased in the phase associated with repeated administration or extended release formulations, with increased exposure time to CPT and its analogues (Lalloo et al., 2004).

Both drug loaded and blank PEI-6-O-CAPRO- $\beta-C D$ nanoparticles showed serious toxicity on cancer cells, but this is thought to be due to the coating polymer PEI rather than the CPT as previously observed in $\mathrm{L} 929$ cells. Therefore, the HT-29 cyctotoxicity of PEI-6-O-CAPRO- $\beta-C D$ formulation, which was found unsafe on 2929 cells, is ignored.

The other formulations have been found to exhibit higher anticancer activity in comparison with the blank formulations and CPT solution at the same time points and concentrations. It has been found that cationic Poly- $\beta-C D-C 6$ nanoparticles showed higher anticancer activity compared to CS-6-O-CAPRO- $\beta$-CD formulations due to the increased cell intake as result of the high cationic property and strong cellular interaction. It has been reported that by Varan et al. the interaction of cationic nanoparticles with negatively charged cell membrane is higher, and this has been shown to be due to interaction with negatively charged molecules in the cell membrane (Varan et al., 2016). Similarly, another study by Verma et al. emphasized the importance of the surface properties and zeta potential of the nanomaterial in the cellular interaction and intracellular mechanisms of nanomaterials (Verma and Stellacci, 2010). It is also known that CDs and self-assembled amphiphilic CD NPs are able to remove cholesterol from the cell membrane and negatively alter membrane dynamics, permeability and endocytic cell uptake mechanisms. In this context, it is know that methylated- $\beta-C D$ is used as an endocytosis inhibitor (Vercauteren et al., 2010). Therefore, $\mathrm{CD}$ interaction with cell membrane constituents, cholesterol in particular, is expected to be stronger with a more amphiphilic and cationic character. In fact, Varan et al also demonstrated that cationic poly- $\beta-C D-C 6$ nanoparticles are able to extract high amounts of cholesterol from MCF-7 breast cancer cells as well as uncoated 6-O-CAPRO- $\beta$-CD nanoparticles inducing apoptosis through a mitochondrial pathway (Varan et al., 2016). 
Formulations of CS-6-O-CAPRO- $\beta-C D$ and Poly- $\beta-C D-C 6$ exhibit higher anticancer activity than equivalent concentrations of camptothecin solution throughout the study. This indicates that the increased therapeutic effect expected from the nano formulation could be achieved. Compared with the drug solution, according to 48 hours data; all nanoparticles at a concentration of $0.1 \mu \mathrm{g} / \mathrm{mL}$ and $0.5 \mu \mathrm{g} / \mathrm{mL}$ had significantly higher $(p<0.05)$ anticancer activity. Considering cell viability, the most suitable dose dependent profile was obtained with Poly- $\beta-C D-C 6$ nanoparticles and it showed significantly higher anticancer activity at all concentrations and time points compared to the drug solution. The results show that by encapsulating CPT into amphiphilic CD nanoparticles, higher anticancer activity could be achieved than when camptothecin is used as an oral solution. Increased anticancer activity depends on the degree of cellular interaction and uptake of the drug delivery system as results of the characterization parameters such as surface charge and particle size (He et al., 2010).

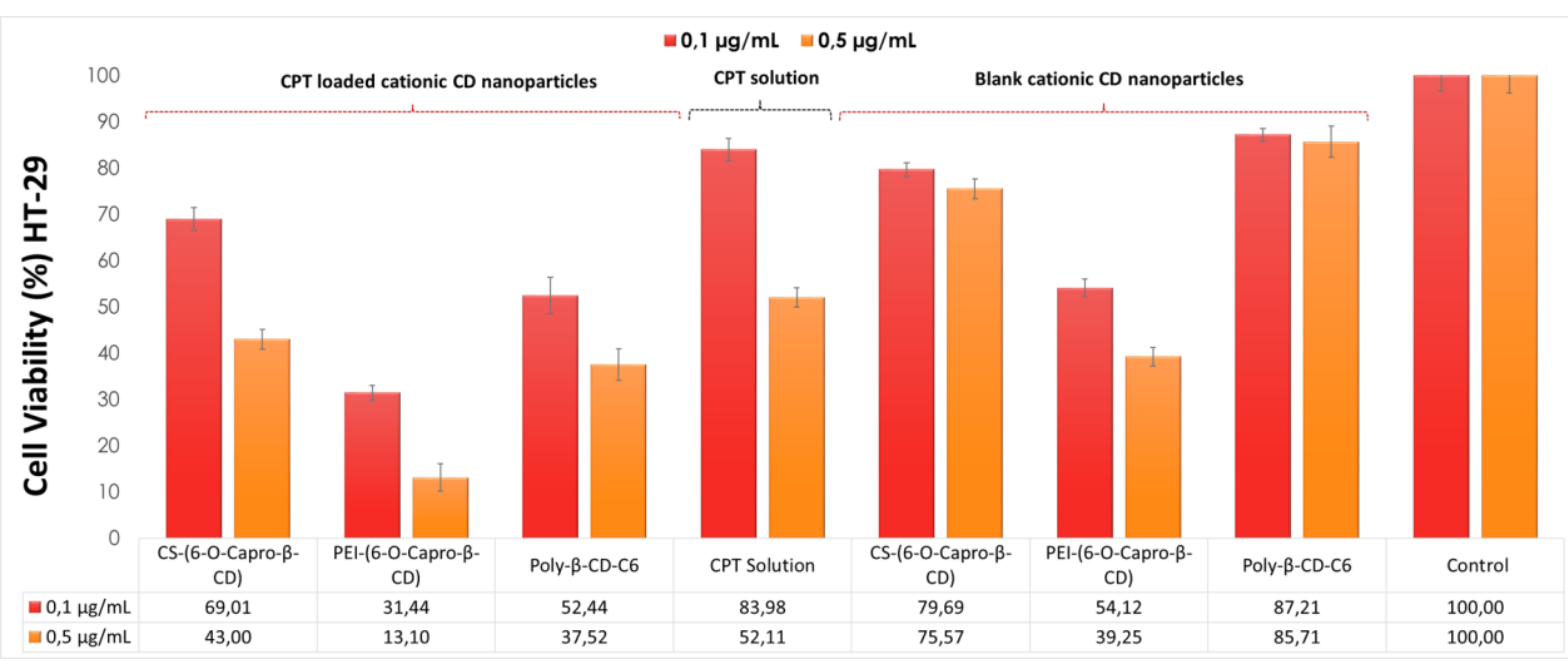

Figure 7. Anticancer activity of CPT-loaded and blank amphiphilic CD nanoparticle formulations and CPT solution in DMSO on HT-29 cell line after $48 \mathrm{~h}$ of incubation $(n=3, \pm S D)$.

\section{In vitro transport evaluation of CPT loaded CD nanoparticles across Caco-2 cell line}

The permeability of the oral formulations is a crucial parameter for bioavailability in terms of their ability to penetrate the intestinal lumen and act on the tissues and pass into the systemic circulation. The ability of the monolayer cell structure formed by the Caco-2 cell line on the transwell to mimic the epithelial cell layer in the intestinal lumen depends on the robustness of the resulting cell layer and the transepithelial electrical resistance (TEER) . 
Intestinal permeability of free CPT and CPT-loaded into nanoparticles was investigated on the Caco-2 cell line. The apparent permeability coefficient $\left(P_{a p p}\right)$ of free CPT and nanoparticulate CPT formulations was found to decrease in the order of CS-(6-O-CAPRO- $\beta$ CD) > Poly- $\beta-C D-C 6>$ PEI-(6-O-Capro-B-CD) > CPT. The $P_{\text {app }}$ of all nanoparticle formulations were significantly different compared to the CPT solution and each other, and significantly higher than the CPT solution $(p<0.05)$.

$P_{\text {app }}$ increased 2.9, 2.76, 2.16 and 1.64 folds for CS-6-O-CAPRO- $\beta-C D$, Poly- $\beta-C D-C 6,6-O-$ CAPRO- $\beta-C D$ and PEI-6-O-Capro-B-CD nanoparticles in comparison to the permeability of the free drug CPT. It was considered that obtaining the highest apparent permeability coefficient in CS-6-O-CAPRO- $\beta$-CD nanoparticles was related to increased cellular interaction due to the positive charge gained by modification of the nanoparticle surface with CS and increased adhesion to the cell and the residence time on the cell. When considered in this context, an increase of 2.76 fold comparison to the permeability constant of the free drug CPT was observed in the second highest formulation Poly- $\beta-C D-C 6$, which could provide the increase in permeability, and it was accepted as another promising formulation in terms of results. On the basis of this increase in drug permeability, it has been contemplated that the average particle size of Poly- $\beta-C D-C 6$ nanoparticles, which is smaller than other formulations, may result in increased permeability from the intestinal cell layer.

Unal et al. investigated the permeabilities of the anionic and cationic CD nanoparticles loaded with the CPT from the Caco-2 cell layer, it was reported that a 50\% increase in the permeability from the Caco-2 cell layer was achieved compared to the anionic form of the nanoparticles (Ünal et al., 2015b). Also, Derakhshandeh et al. investigated the effect of particle size of nanoparticles on the permeability of the Caco-2 cell layer. For this purpose, CPT loaded PLGA nanoparticles with different particle sizes were prepared and their permeability was investigated. The results showed that by increasing the particle size gradually from $100 \mathrm{~nm}$ to $950 \mathrm{~nm}$, the permeability of the CPT loaded PLGA nanoparticles from the Caco-2 cell layer decreased (Derakhshandeh et al., 2011).

It is also known that there is a transition from the active lactone form to the inactive carboxylate form of CPT at neutral and basic pHs in physiological environment, and diffusion of the inactive form from the biological membranes is slower and lower than the active lactone form (Thakral et al., 2012). At pH 7.4, in which permeability studies are carried out, 
the free camptothecin active lactone form is known to gradually hydrolyse into the inactive carboxylate form. In this context, Poly- $\beta-C D-C 6$ formulation, which exhibits a slower release than other formulations and diffuses faster and easily through the intestinal cell barrier due to its smaller particle size, might provide more stable and effective permeation of camptothecin through the intestinal cell layer. When all the information were taken into consideration, it was possible to prevent the hydrolysis of camptothecin in an encapsulated system from the physiological conditions and to increase its stability by slowing the conversion to inactive carboxylate form. Thus, it has been found that camptothecin, which can be preserved in its active lactone form, may diffuse more through the intestinal cell layer than its inactive form, the carboxylate form (Yue et al., 2011).

Table 3. Apparent permeability coefficient $\left(P_{\text {aap }}\right)$ of different CPT loaded CD nanoparticle formulations $(n=3, \pm$ $\mathrm{SD})$.

\begin{tabular}{|c|c|c|}
\hline Formulations & $\mathrm{P}_{\mathrm{app}}\left(\times 10^{-6} \mathrm{~cm} / \mathrm{s}\right) \pm \mathrm{SD}$ & Increase in permeability (\%) \\
\hline Free CPT & $3.12 \pm 0.49$ & - \\
\hline CS-(6-O-CAPRO- $\beta-C D)$ & $9.15 \pm 1.02$ & 293.2 \\
\hline PEI-(6-O-CAPRO- $\beta-C D)$ & $5.13 \pm 1.27$ & 164,4 \\
\hline Poly- $\beta-C D-C 6$ & $8.64 \pm 1.31$ & 276.9 \\
\hline
\end{tabular}

\section{Conclusion and Future Perspectives}

In today's knowledge, pathophysiology related to many diseases have been enlightened to a great extent and, accordingly, new forms of treatments have emerged. Drug administration by injection reduces the effectiveness of treatment during the procedure due to patient compliance, severity and frequency of side effects, and difficulty in administration. Therefore, as an alternative to parenteral administration, transdermal, nasal, buccal, pulmonary or oral administration methods are being studied. Oral administration is preferred among all these alternatives. In terms of ease of application and patient compliance, it stands out as the most advantageous way to be used in chronic diseases. Orally administered drugs can be designed to treat gastrointestinal tract (GIT) diseases locally or to achieve a systemic effect by passing into the bloodstream. In addition to the 
advantages provided by oral drug administration, there are some obstacles that must be overcome when evaluated in terms of pharmaceutical science. Today, orally administered drugs, which make up the majority of the pharmaceutical market, are growing rapidly in line with the increasing demand in parallel with the benefits provided by the patient in practice. In line with the demand in question, scientific studies regarding the drug administration by the oral route continue increasingly.

Localized cancer therapy facilitates the delivery of chemo- or immunotherapeutic- drug directly to the site of disease in a controlled manner, sparing off-target tissue toxicities. Colon specific drug delivery systems are intended for local treatment of colon site specific diseases such as ulcerative colitis (UC), irritable bowel disease (IBD) and CRC, or for the peptide-protein drugs that are intended to be absorbed at the colon. Various formulation approaches have been developed for drug delivery to the colon. These are in principle approaches that aim to deliver drugs to the colon region by making use of one or more of the physiological properties of the GIT. The fact that different segments of the GIT are in different $\mathrm{pH}$ ranges, enzymes activity in the GIT and the activities of colonic bacteria present in the colon region are the main conditions used in the development of colon targeted drug delivery systems.

The point reached at the end of the our study; it is possible to perform an oral cationic amphiphilic CD nanoparticulate drug delivery system for the anticancer treatment of colorectal tumors with oral drug delivery systems, which can be used in the treatment of cancer rarely, and specifically not applicable to digestive tract tumors. Thus, a nanoparticulate drug delivery system has been developed that can be administered orally and provides local treatment by delivering the drug it carries to the effect area. For this reason, a nanoparticulate drug delivery system, which can be administered orally but which acts locally, thus preventing or minimizing the systemic side effects of the drug they contain, is aimed at both reducing the side effects and increasing the stability of the drug. In addition, due to the submicron sizes of the nanoparticles, it is considered that the drug concentration in the region can be increased due to the increased permeability and permeability effect, due to the nanoparticle, which can be highly localized in the tumor tissue.

In order to inspire further studies in the future, the findings of our study, that designed a cationic amphiphilic CD nanoparticulate drug delivery system, which is targeted to the colon 
with a passive targeting strategy, can be administered orally, remains stable until the colon, releases the active substance it carries as a result of degradation by the colonic microflora, can be used. In this context, with the development of new amphiphilic cationic CD nanoparticle formulations that can carry active targeting ligands for the colon tumors in the future, it is considered that systems that reach the colon with passive targeting and that offer a more specific treatment approach focused on the tumor with active targeting in the colon.

\section{Acknowledgements}

The authors thank the Spanish Ministry of Science and Innovation (ref RTI2018-097609-BC21) for financial support.

\section{References}

Bandi, S.P., Kumbhar, Y.S., Venuganti, V.V.K., 2020. Effect of particle size and surface charge of nanoparticles in penetration through intestinal mucus barrier. Journal of Nanoparticle Research 22, 1-11.

Belali, N., Wathoni, N., Muchtaridi, M., 2019. Advances in orally targeted drug delivery to colon. Journal of advanced pharmaceutical technology \& research 10, 100.

Bilensoy, E., 2010. Cationic nanoparticles for cancer therapy. Expert opinion on drug delivery 7, 795809.

Bilensoy, E., Gürkaynak, O., Doğan, A.L., Hıncal, A.A., 2008. Safety and efficacy of amphiphilic ßcyclodextrin nanoparticles for paclitaxel delivery. International journal of pharmaceutics 347, 163 170.

Bilensoy, E., Hincal, A.A., 2009. Recent advances and future directions in amphiphilic cyclodextrin nanoparticles. Expert opinion on drug delivery 6, 1161-1173.

Borges, O., Borchard, G., Verhoef, J.C., de Sousa, A., Junginger, H.E., 2005. Preparation of coated nanoparticles for a new mucosal vaccine delivery system. International journal of pharmaceutics 299 , 155-166.

Chao, J., Lin, J., Frankel, P., Clark, A.J., Wiley, D.T., Garmey, E., Fakih, M., Lim, D., Chung, V., Luevanos, E., 2017. Pilot trial of CRLX101 in patients with advanced, chemotherapy-refractory gastroesophageal cancer. Journal of gastrointestinal oncology 8, 962.

Date, A.A., Hanes, J., Ensign, L.M., 2016. Nanoparticles for oral delivery: Design, evaluation and stateof-the-art. Journal of Controlled Release 240, 504-526.

Davis, M.E., 2009. Design and development of IT-101, a cyclodextrin-containing polymer conjugate of camptothecin. Advanced drug delivery reviews 61, 1189-1192.

Derakhshandeh, K., Hochhaus, G., Dadashzadeh, S., 2011. In-vitro cellular uptake and transport study of 9-nitrocamptothecin PLGA nanoparticles across Caco-2 cell monolayer model. Iranian journal of pharmaceutical research: IJPR 10, 425.

Díaz-Moscoso, A., Balbuena, P., Gómez-García, M., Mellet, C.O., Benito, J.M., Le Gourriérec, L., Di Giorgio, C., Vierling, P., Mazzaglia, A., Micali, N., 2008. Rational design of cationic cyclooligosaccharides as efficient gene delivery systems. Chemical communications, 2001-2003. 
Díaz-Moscoso A, Le Gourriérec L, Gómez-García M, Benito JM, Balbuena P, Ortega-Caballero F, Guilloteau N, Di Giorgio C, Vierling P, Defaye J, Ortiz Mellet C, García Fernández JM. 2009. Polycationic amphiphilic cyclodextrins for gene delivery: synthesis and effect of structural modifications on plasmid DNA complex stability, cytotoxicity, and gene expression. Chemistry. 15(46):12871-88.

Erdogar, N., Varan, G., Bilensoy, E., 2017. Amphiphilic cyclodextrin derivatives for targeted drug delivery to tumors. Current topics in medicinal chemistry 17, 1521-1528.

Fallingborg, J., 1999. Intraluminal pH of the human gastrointestinal tract. Danish medical bulletin 46, 183-196.

Forgue-Lafitte, M.-E., Coudray, A.-M., Bréant, B., Mešter, J., 1989. Proliferation of the human colon carcinoma cell line HT29: autocrine growth and deregulated expression of the c-myc oncogene. Cancer research 49, 6566-6571.

Gao, W., Lai, J.C., Leung, S., 2012. Functional enhancement of chitosan and nanoparticles in cell culture, tissue engineering, and pharmaceutical applications. Frontiers in physiology 3, 321.

Griffin, W.C., 1949. Classification of surface-active agents by" HLB". J. Soc. Cosmet. Chem. 1, 311-326. Griffin, W.C., 1954. Calculation of HLB values of non-ionic surfactants. J. Soc. Cosmet. Chem. 5, 249256.

He, C., Hu, Y., Yin, L., Tang, C., Yin, C., 2010. Effects of particle size and surface charge on cellular uptake and biodistribution of polymeric nanoparticles. Biomaterials 31, 3657-3666.

Honary, S., Zahir, F., 2013. Effect of zeta potential on the properties of nano-drug delivery systems-a review (Part 1). Tropical Journal of Pharmaceutical Research 12, 255-264.

Huang, Z.r., Hua, S.c., Yang, Y.I., Fang, J.y., 2008. Development and evaluation of lipid nanoparticles for camptothecin delivery: a comparison of solid lipid nanoparticles, nanostructured lipid carriers, and lipid emulsion. Acta Pharmacologica Sinica 29, 1094-1102.

Jiménez Blanco, J.L., Benito, J.M., Ortiz Mellet, C., García Fernández, J.M. 2017. Molecular nanoparticle-based gene delivery systems . Journal of Drug Delivery Science and Technology, 42, 1837

Jones, P.A., Baylin, S.B., 2007. The epigenomics of cancer. Cell 128, 683-692.

Lai, S.K., Wang, Y.-Y., Hanes, J., 2009. Mucus-penetrating nanoparticles for drug and gene delivery to mucosal tissues. Advanced drug delivery reviews 61, 158-171.

Lalloo, A.K., Luo, F.R., Guo, A., Paranjpe, P.V., Lee, S.-H., Vyas, V., Rubin, E., Sinko, P.J., 2004. Membrane transport of camptothecin: facilitation by human P-glycoprotein (ABCB1) and multidrug resistance protein 2 ( $A B C C 2$ ). BMC medicine 2, 16.

Lazzari, S., Moscatelli, D., Codari, F., Salmona, M., Morbidelli, M., Diomede, L., 2012. Colloidal stability of polymeric nanoparticles in biological fluids. Journal of nanoparticle research 14, 920.

Loftsson, T., Brewster, M.E., 2011. Pharmaceutical applications of cyclodextrins: effects on drug permeation through biological membranes. Journal of Pharmacy and Pharmacology 63, 1119-1135.

Luo, C., Sun, J., Du, Y., He, Z., 2014. Emerging integrated nanohybrid drug delivery systems to facilitate the intravenous-to-oral switch in cancer chemotherapy. Journal of controlled release 176, 94-103.

Mazzaferro, S., Bouchemal, K., Skanji, R., Gueutin, C., Chacun, H., Ponchel, G., 2012. Intestinal permeation enhancement of docetaxel encapsulated into methyl- $\beta$-cyclodextrin/poly (isobutylcyanoacrylate) nanoparticles coated with thiolated chitosan. Journal of controlled release 162, 568-574.

Memişoğlu, E., Bochot, A., Özalp, M., Şen, M., Duchêne, D., Hincal, A.A., 2003. Direct formation of nanospheres from amphiphilic $\beta$-cyclodextrin inclusion complexes. Pharmaceutical research $20,117-$ 125.

Memişoğlu, E., Bochot, A., Şen, M., Charon, D., Duchêne, D., Hıncal, A.A., 2002. Amphiphilic $\beta$ cyclodextrins modified on the primary face: synthesis, characterization, and evaluation of their 
potential as novel excipients in the preparation of nanocapsules. Journal of pharmaceutical sciences 91, 1214-1224.

Minelli, R., Cavalli, R., Ellis, L., Pettazzoni, P., Trotta, F., Ciamporcero, E., Barrera, G., Fantozzi, R., Dianzani, C., Pili, R., 2012. Nanosponge-encapsulated camptothecin exerts anti-tumor activity in human prostate cancer cells. European journal of pharmaceutical sciences 47, 686-694.

Mohanraj, V., Chen, Y., 2006. Nanoparticles-a review. Tropical journal of pharmaceutical research 5, 561-573.

Mora-Huertas, C.E., Fessi, H., Elaissari, A., 2010. Polymer-based nanocapsules for drug delivery. International journal of pharmaceutics 385, 113-142.

Okon, E.U., Hammed, G., El Wafa, P.A., Abraham, O., Case, N., Henry, E., 2014. In-vitro cytotoxicity of Polyethyleneimine on HeLa and Vero Cells. International Journal of Innovation and Applied Studies 5, 192.

Pflueger, I., Charrat, C., Mellet, C.O., Fernández, J.M.G., Di Giorgio, C., Benito, J.M., 2016. Cyclodextrin-based facial amphiphiles: assessing the impact of the hydrophilic-lipophilic balance in the self-assembly, DNA complexation and gene delivery capabilities. Organic \& biomolecular chemistry 14, 10037-10049.

Plapied, L., Duhem, N., des Rieux, A., Préat, V., 2011. Fate of polymeric nanocarriers for oral drug delivery. Current opinion in colloid \& interface science 16, 228-237.

Qiu, N., Li, X., Liu, J., 2017. Application of cyclodextrins in cancer treatment. Journal of Inclusion Phenomena and Macrocyclic Chemistry 89, 229-246.

Reul, R., Renette, T., Bege, N., Kissel, T., 2011. Nanoparticles for paclitaxel delivery: a comparative study of different types of dendritic polyesters and their degradation behavior. International journal of pharmaceutics 407, 190-196.

Salatin, S., Barar, J., Barzegar-Jalali, M., Adibkia, K., Kiafar, F., Jelvehgari, M., 2017. Development of a nanoprecipitation method for the entrapment of a very water soluble drug into Eudragit RL nanoparticles. Research in pharmaceutical sciences 12, 1.

Tan, T.B., Yussof, N.S., Abas, F., Mirhosseini, H., Nehdi, I.A., Tan, C.P., 2016. Forming a lutein nanodispersion via solvent displacement method: the effects of processing parameters and emulsifiers with different stabilizing mechanisms. Food chemistry 194, 416-423.

Thakral, N.K., Ray, A.R., Bar-Shalom, D., Eriksson, A.H., Majumdar, D.K., 2012. Soluplus-solubilized citrated camptothecin-a potential drug delivery strategy in colon cancer. Aaps Pharmscitech 13, 5966.

Trapani, A., Lopedota, A., Franco, M., Cioffi, N., leva, E., Garcia-Fuentes, M., Alonso, M.J., 2010. A comparative study of chitosan and chitosan/cyclodextrin nanoparticles as potential carriers for the oral delivery of small peptides. European Journal of Pharmaceutics and Biopharmaceutics 75, 26-32.

Ungaro, F., d'Angelo, I., Coletta, C., di Villa Bianca, R.d.E., Sorrentino, R., Perfetto, B., Tufano, M.A., Miro, A., La Rotonda, M.I., Quaglia, F., 2012. Dry powders based on PLGA nanoparticles for pulmonary delivery of antibiotics: modulation of encapsulation efficiency, release rate and lung deposition pattern by hydrophilic polymers. Journal of controlled release 157, 149-159.

Ünal, H., d’Angelo, I., Pagano, E., Borrelli, F., Izzo, A., Ungaro, F., Quaglia, F., Bilensoy, E., 2015a. Core-shell hybrid nanocapsules for oral delivery of camptothecin: formulation development, in vitro and in vivo evaluation. Journal of Nanoparticle Research 17, 42.

Ünal, H., Öztürk, N., Bilensoy, E., 2015b. Formulation development, stability and anticancer efficacy of core-shell cyclodextrin nanocapsules for oral chemotherapy with camptothecin. Beilstein journal of organic chemistry 11, 204-212.

Varan, G., Benito, J.M., Mellet, C.O., Bilensoy, E., 2017a. Development of polycationic amphiphilic cyclodextrin nanoparticles for anticancer drug delivery. Beilstein journal of nanotechnology 8, 14571468.

Varan, G., Öncül, S., Ercan, A., Benito, J.M., Mellet, C.O., Bilensoy, E., 2016. Cholesterol-targeted anticancer and apoptotic effects of anionic and polycationic amphiphilic cyclodextrin nanoparticles. Journal of pharmaceutical sciences 105, 3172-3182. 
Varan, G., Varan, C., Erdoğar, N., Hıncal, A.A., Bilensoy, E., 2017b. Amphiphilic cyclodextrin nanoparticles. International journal of pharmaceutics 531, 457-469.

Vercauteren, D., Vandenbroucke, R.E., Jones, A.T., Rejman, J., Demeester, J., De Smedt, S.C., Sanders, N.N., Braeckmans, K., 2010. The use of inhibitors to study endocytic pathways of gene carriers: optimization and pitfalls. Molecular Therapy 18, 561-569.

Verma, A., Stellacci, F., 2010. Effect of surface properties on nanoparticle-cell interactions. small 6, $12-21$.

Warner, D.L., Burke, T.G., 1997. Simple and versatile high-performance liquid chromatographic method for the simultaneous quantitation of the lactone and carboxylate forms of camptothecin anticancer drugs. Journal of Chromatography B: Biomedical Sciences and Applications 691, 161-171.

You, X., Kang, Y., Hollett, G., Chen, X., Zhao, W., Gu, Z., Wu, J., 2016. Polymeric nanoparticles for colon cancer therapy: overview and perspectives. Journal of Materials Chemistry B 4, 7779-7792.

Yue, Z.-G., Wei, W., Lv, P.-P., Yue, H., Wang, L.-Y., Su, Z.-G., Ma, G.-H., 2011. Surface charge affects cellular uptake and intracellular trafficking of chitosan-based nanoparticles. Biomacromolecules 12 , 2440-2446.

Zhang, Z., Feng, S.-S., 2006. The drug encapsulation efficiency, in vitro drug release, cellular uptake and cytotoxicity of paclitaxel-loaded poly (lactide)-tocopheryl polyethylene glycol succinate nanoparticles. Biomaterials 27, 4025-4033.

Zunino, F., Dallavalle, S., Laccabue, D., Beretta, G., Merlini, L., Pratesi, G., 2002. Current status and perspectives in the development of camptothecins. Current pharmaceutical design 8, 2505-2520. 


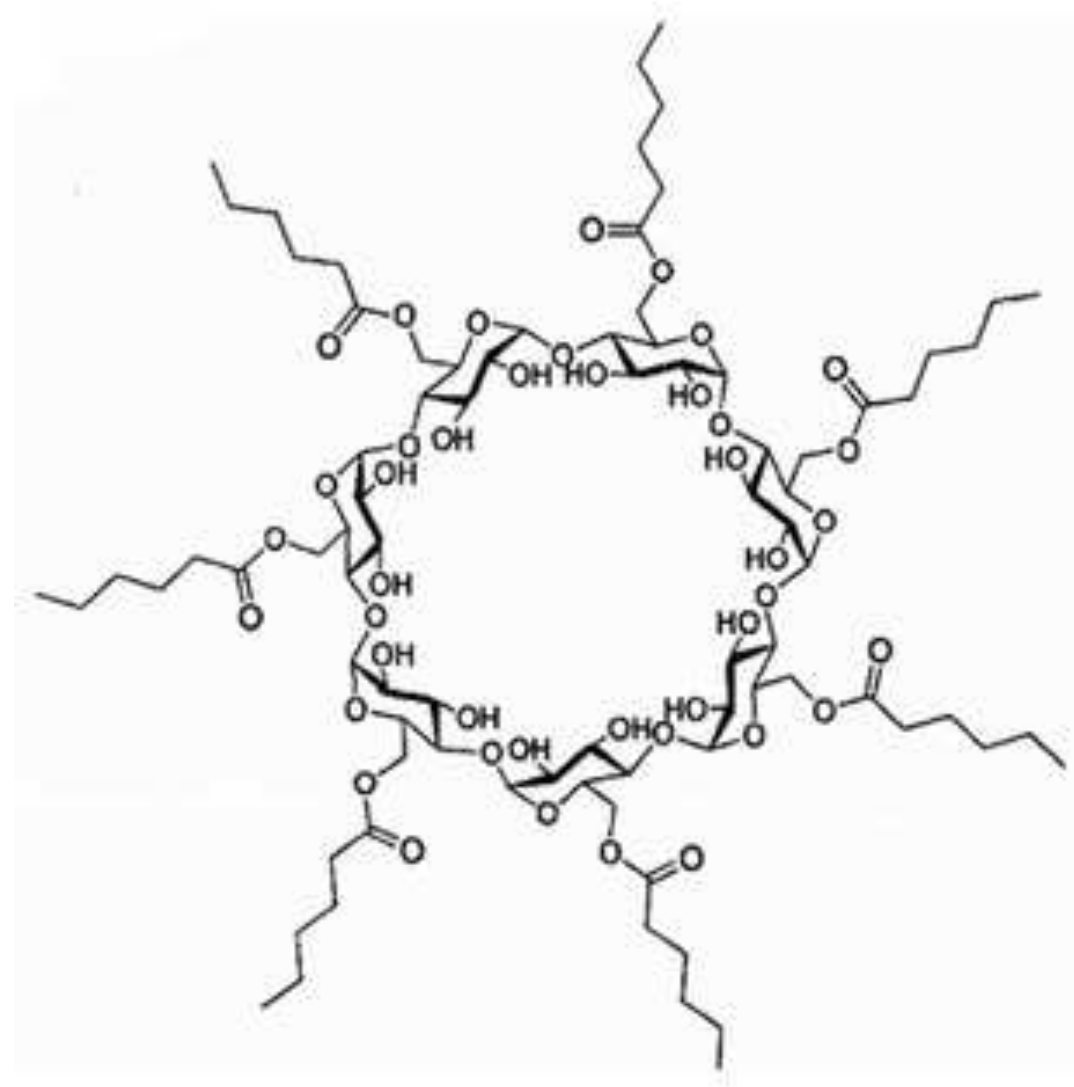

$\mathrm{C}_{84} \mathrm{H}_{140} \mathrm{O}_{42}$

6-O-Capro- $\beta$-CD

MW: $1813 \mathrm{~g} / \mathrm{mol}$

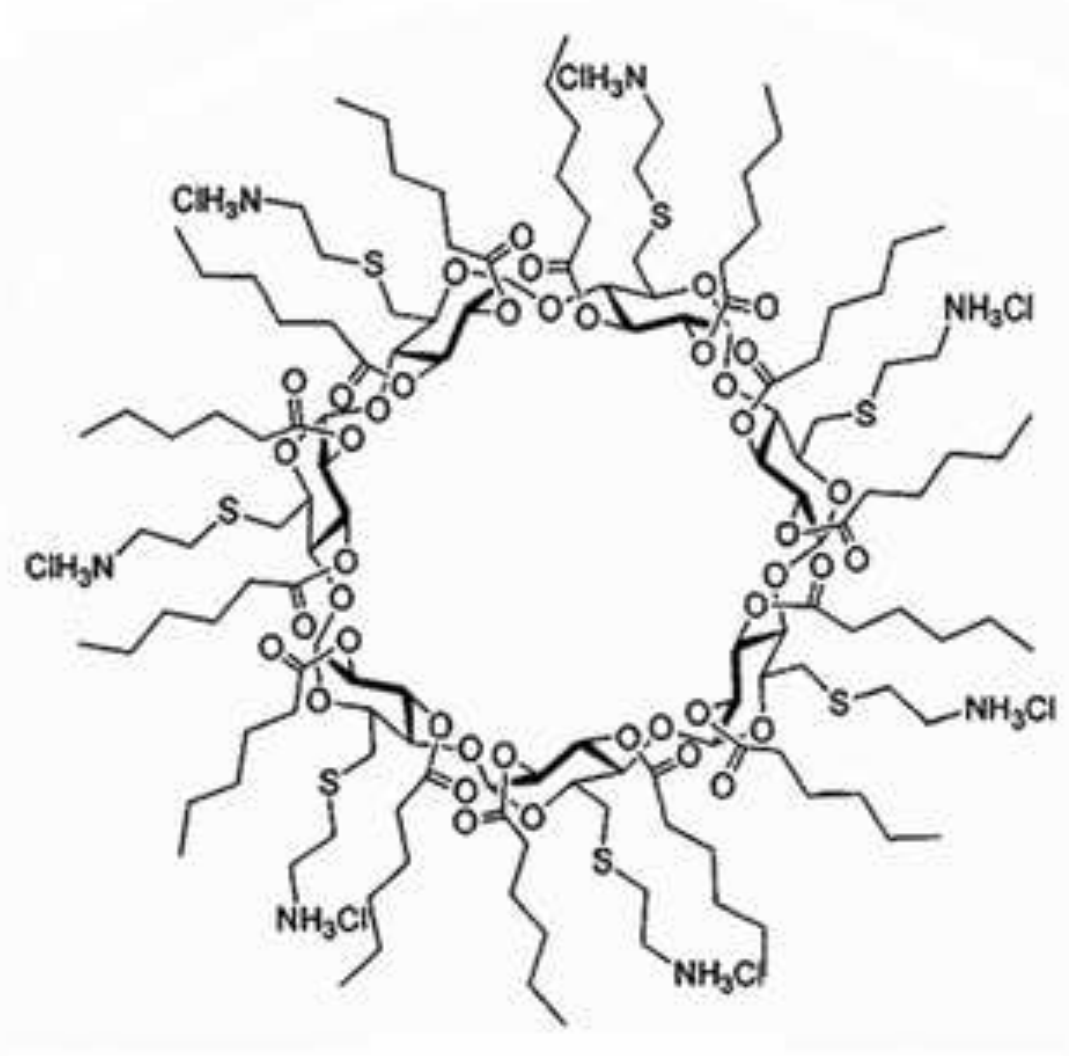

$\mathrm{C}_{140} \mathrm{H}_{252} \mathrm{Cl}_{7} \mathrm{~N}_{7} \mathrm{O}_{42} \mathrm{~S}_{7}$ Poly- $\beta-C D-C 6$

MW: $3178.15 \mathrm{~g} / \mathrm{mol}$ 


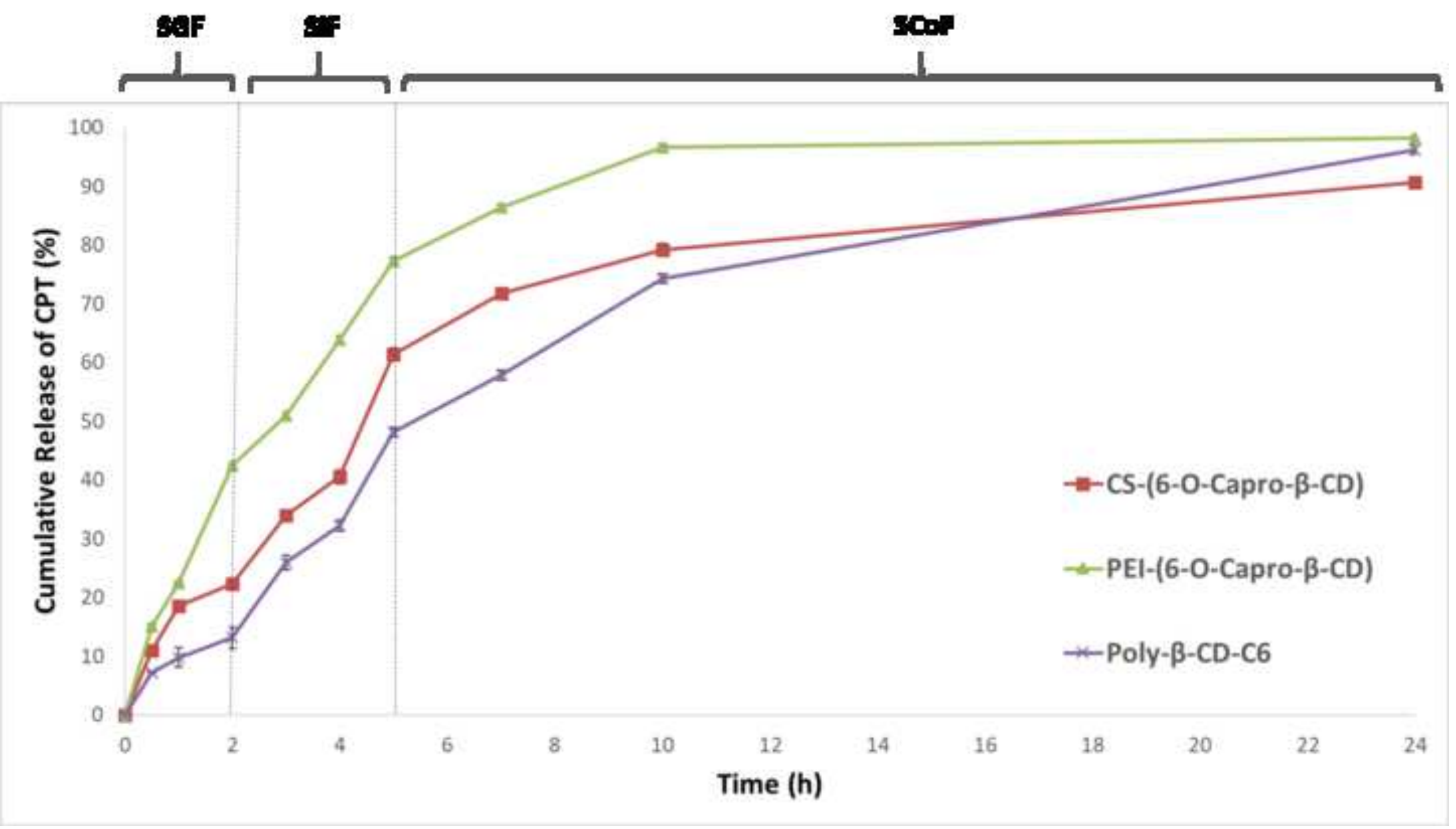




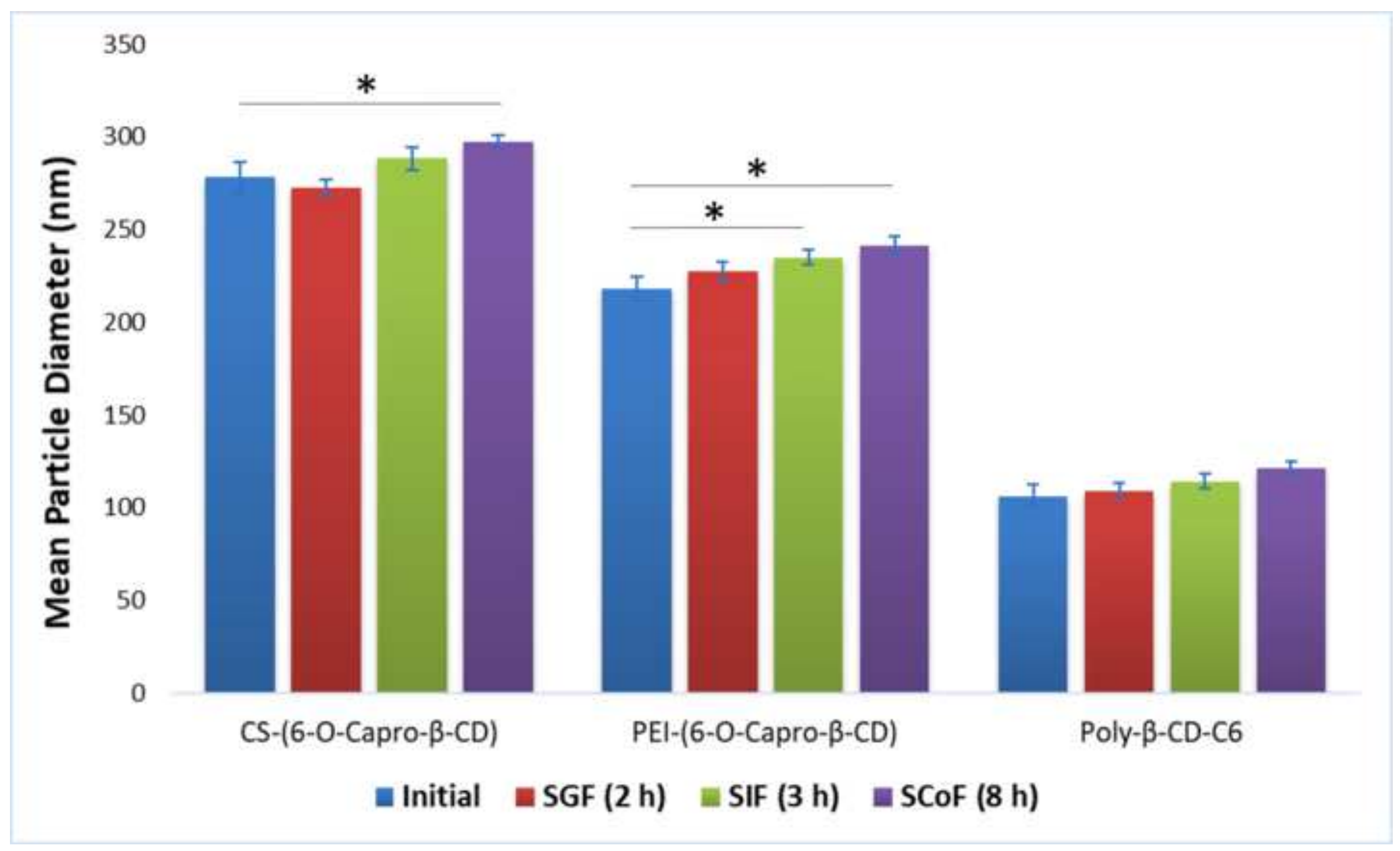




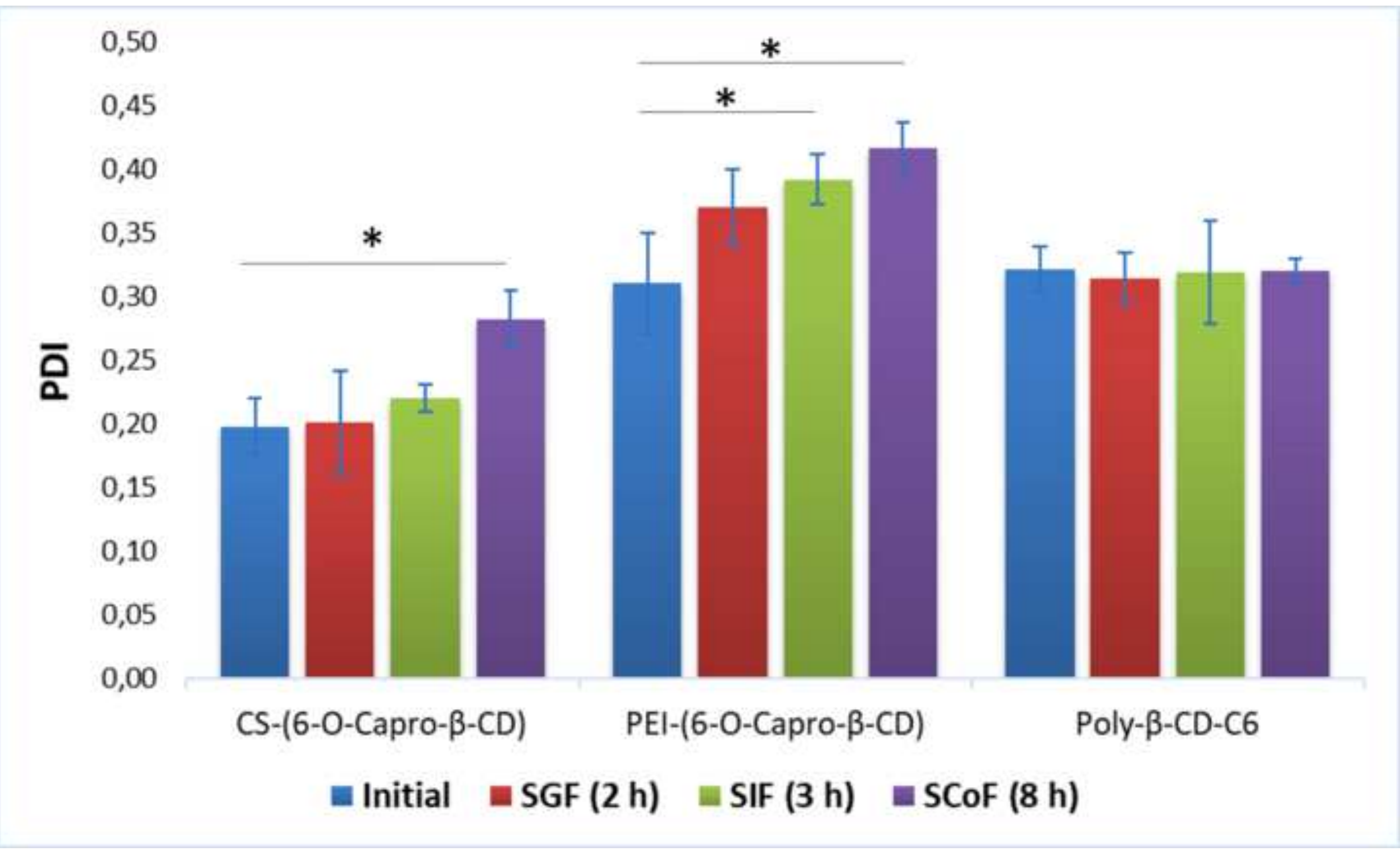




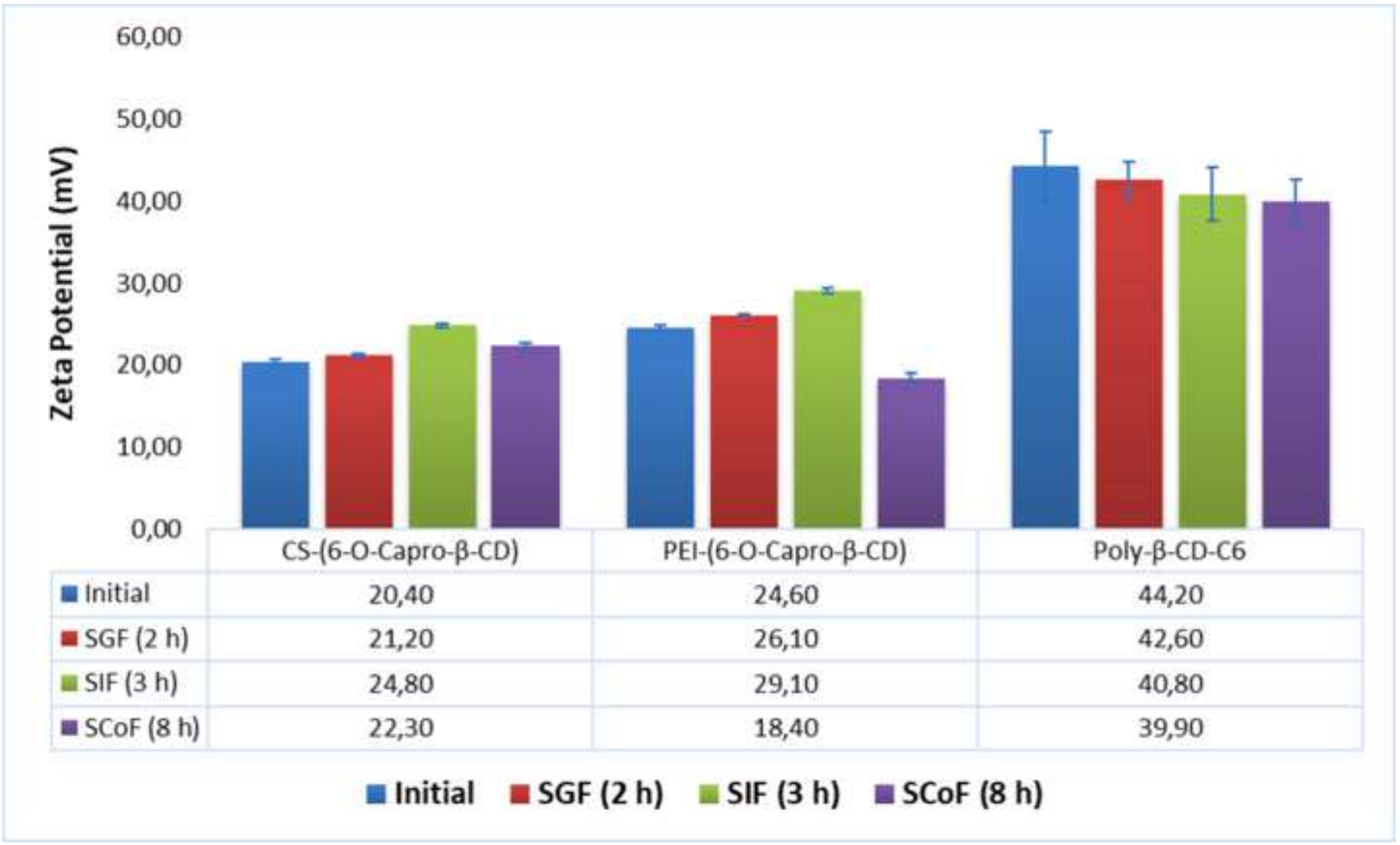




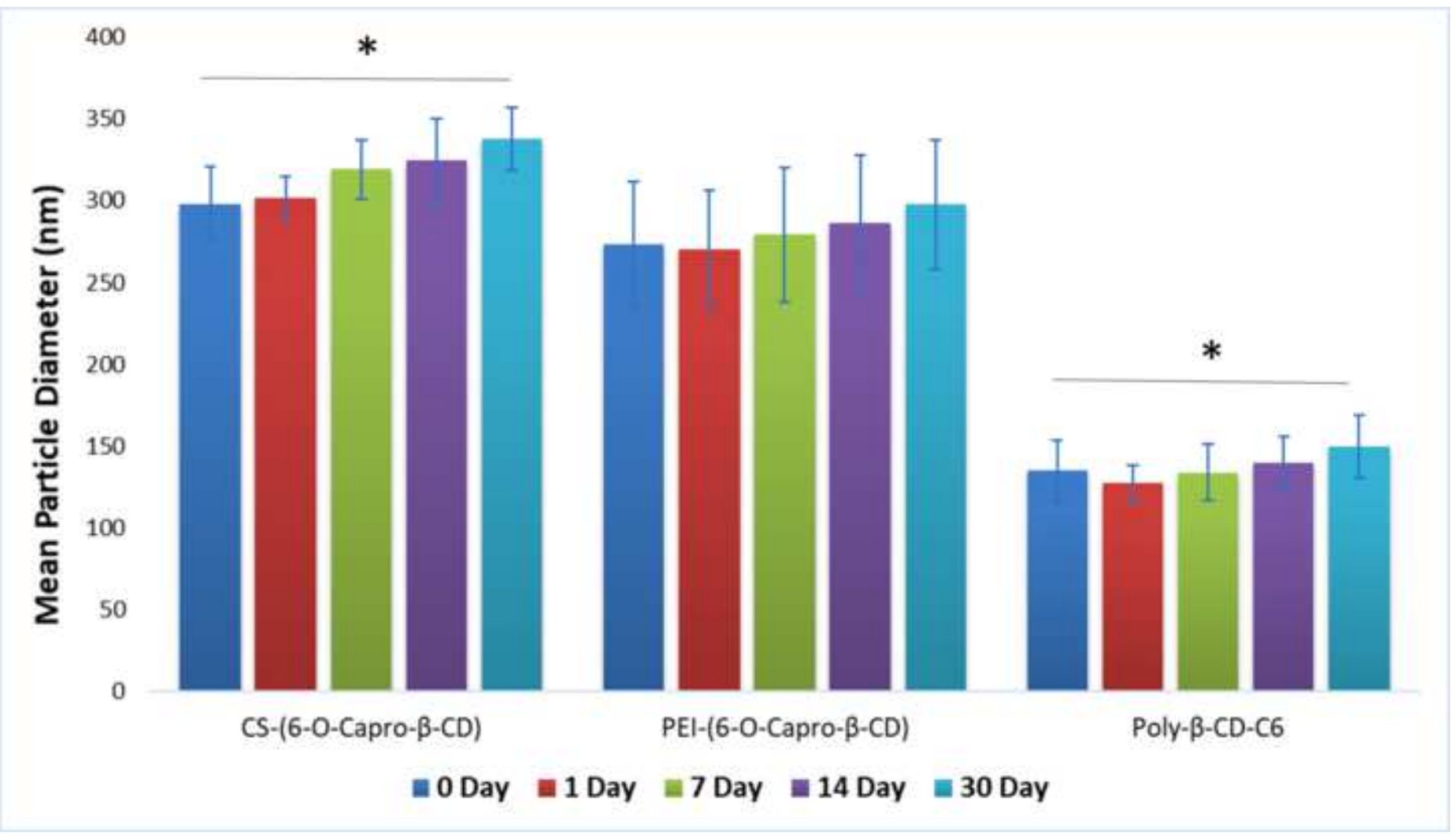




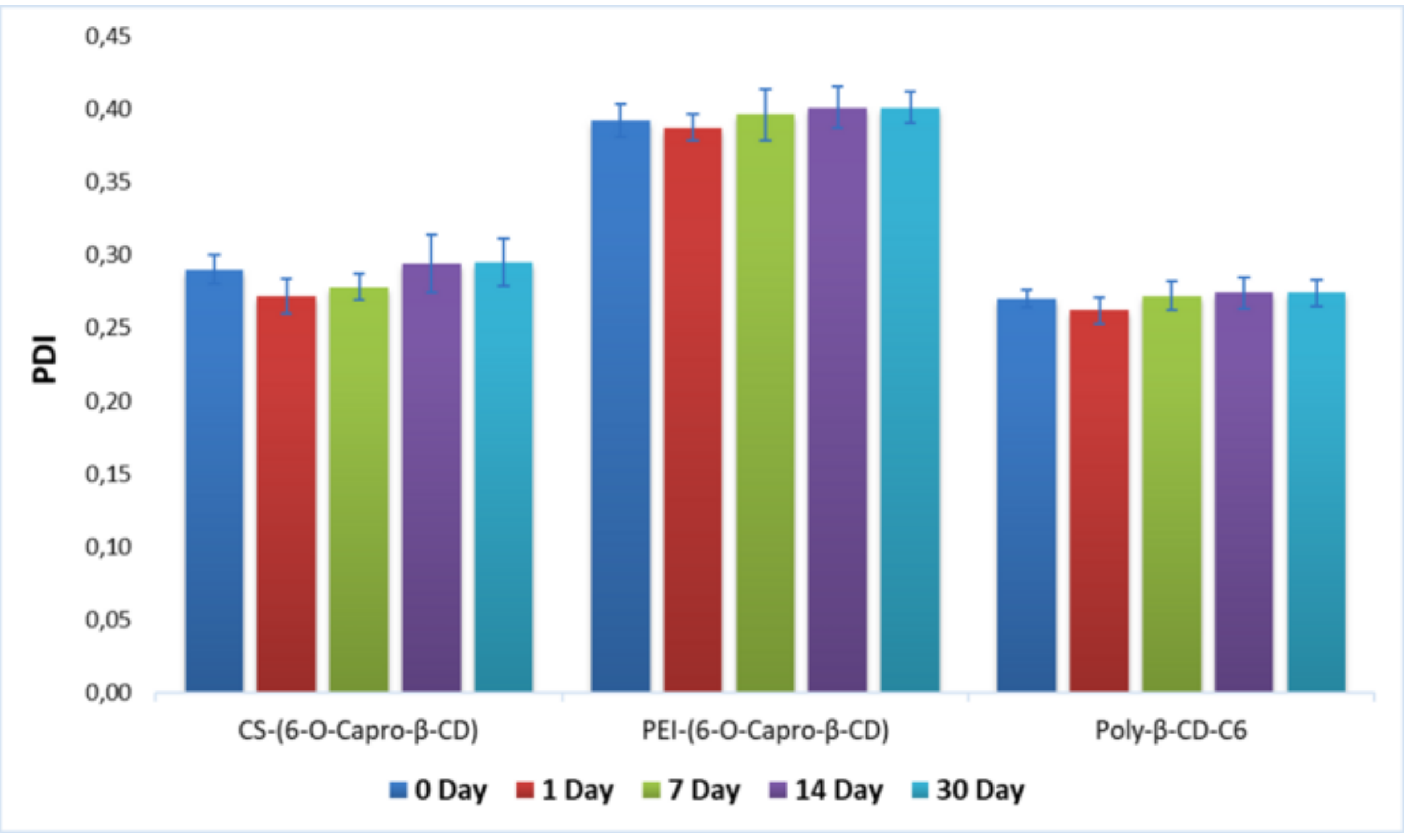




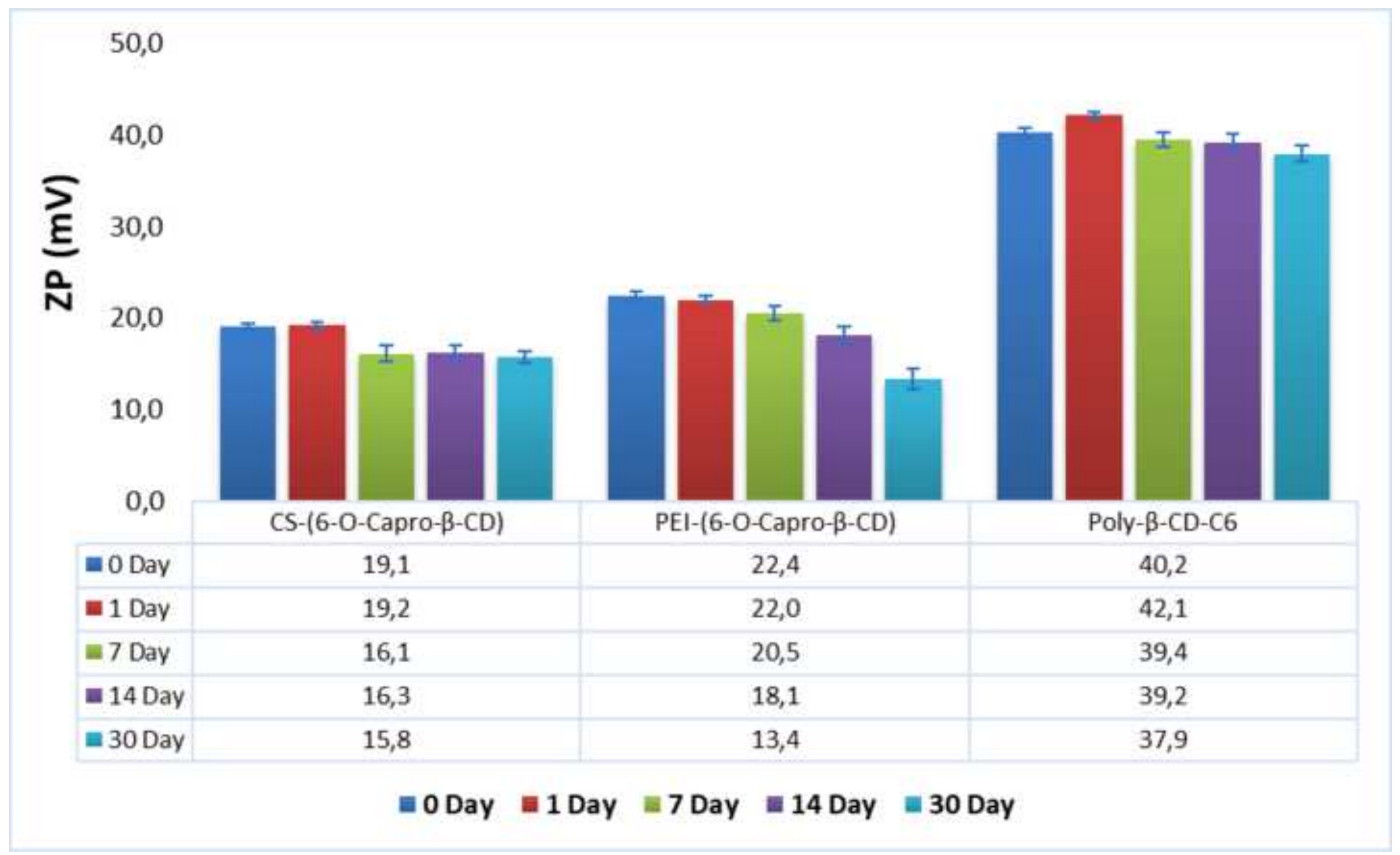

$\sum_{20,0}$
हิ

10,0

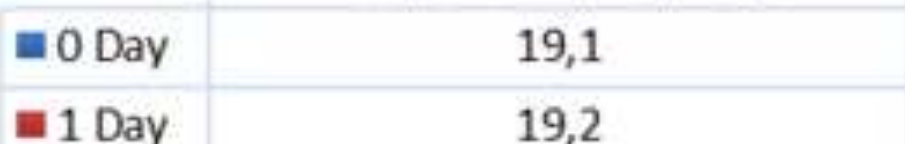

=7 Day

14 Day

16,3

15,8

EDay 1 Day 


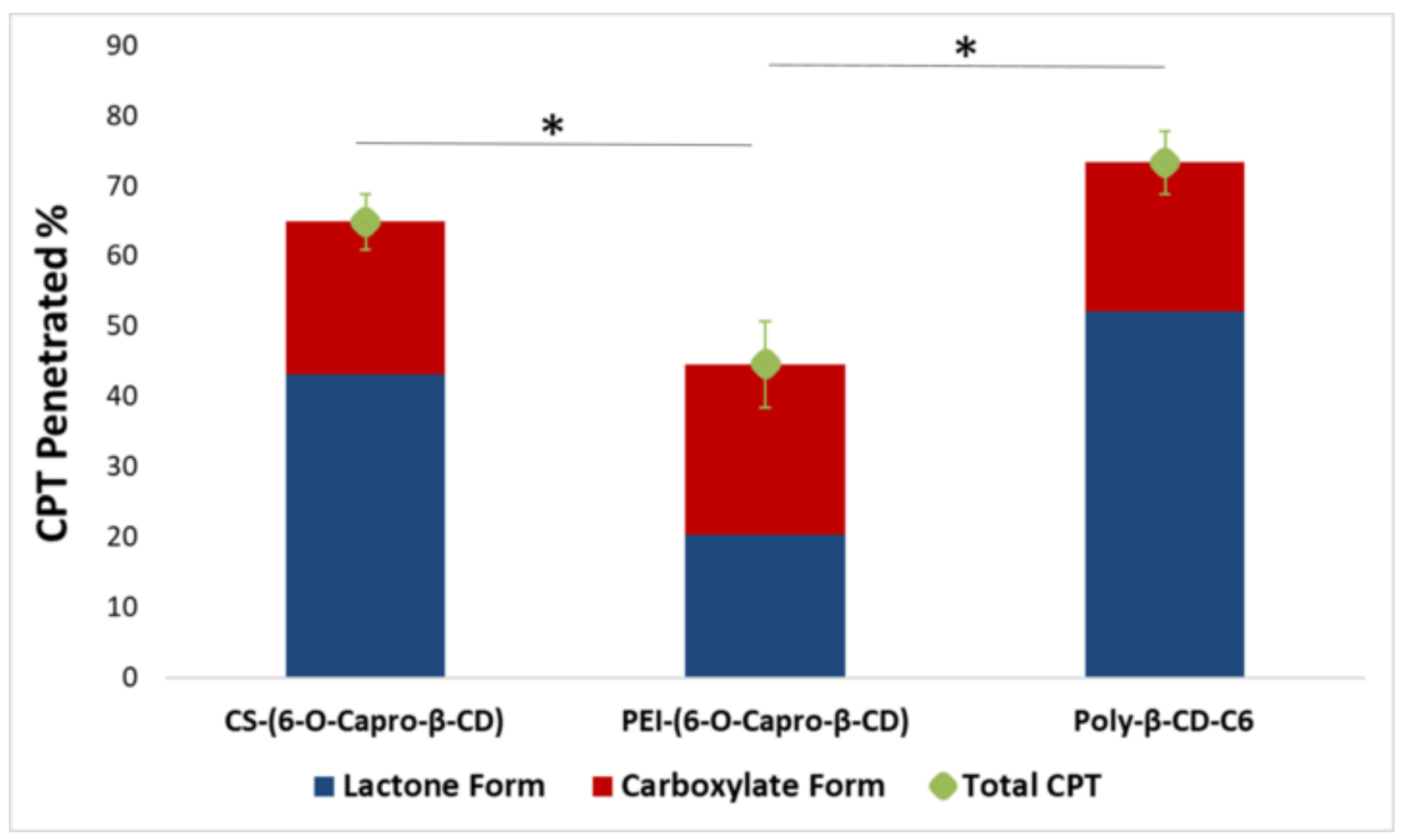




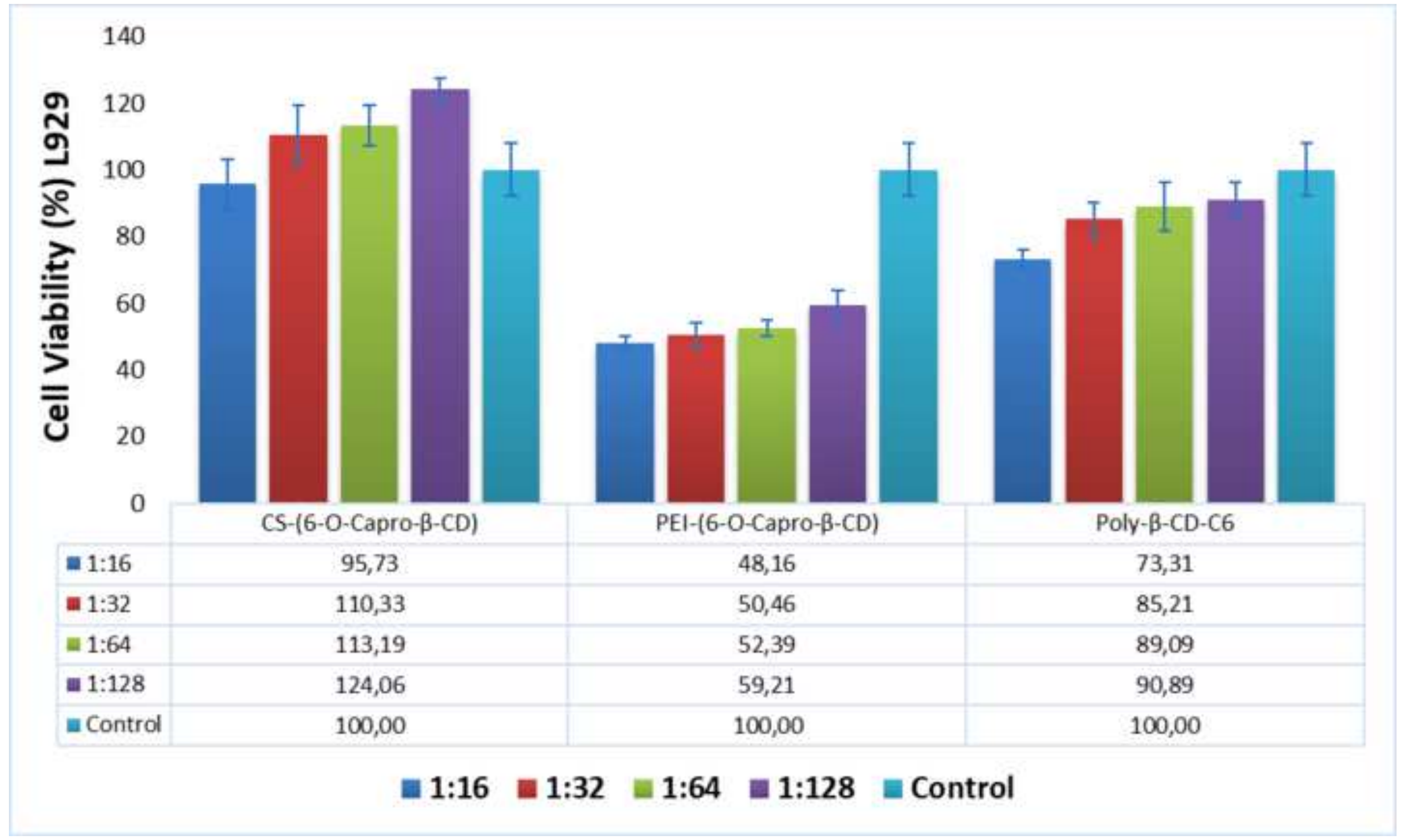




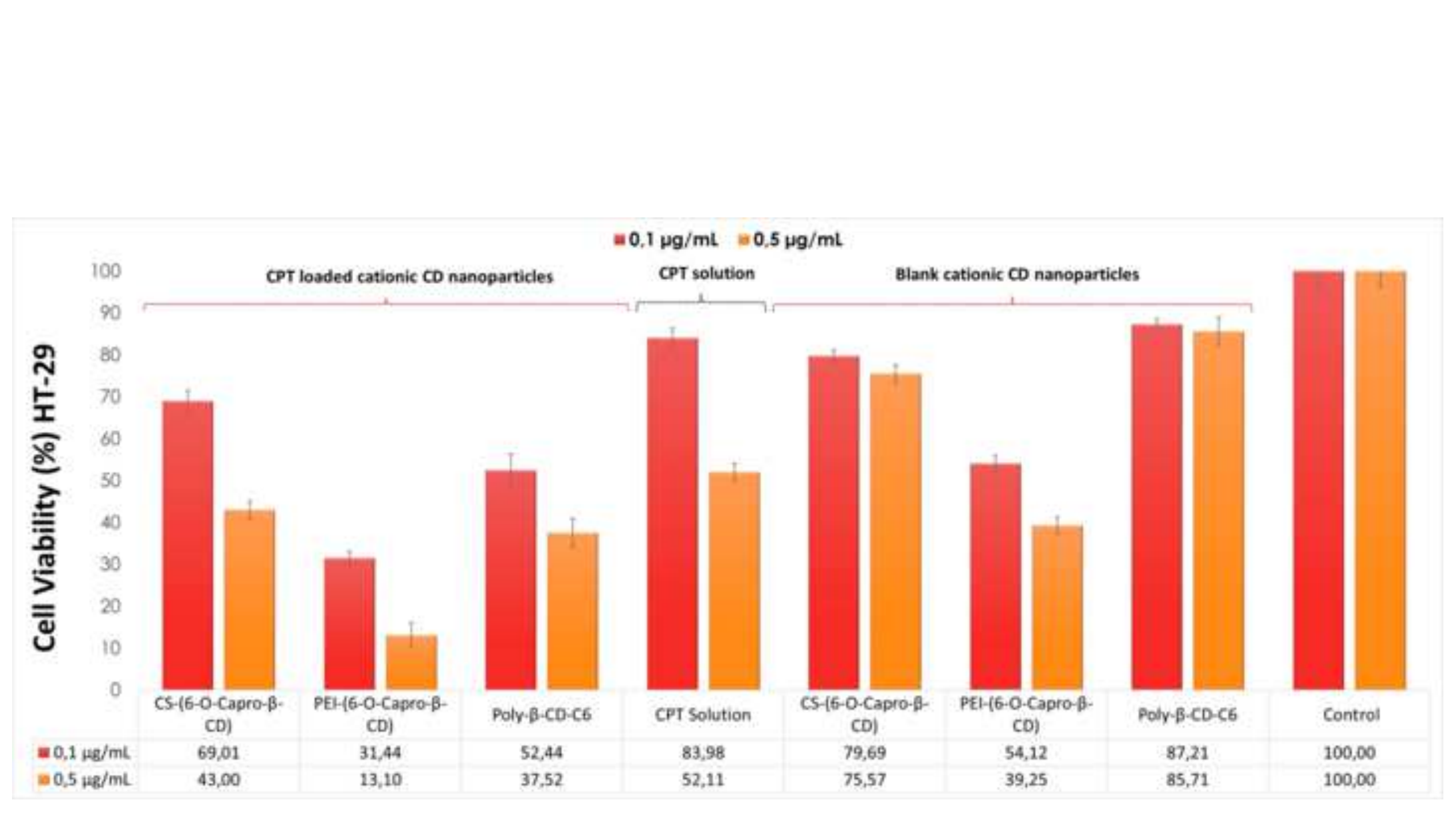

Figure(s)

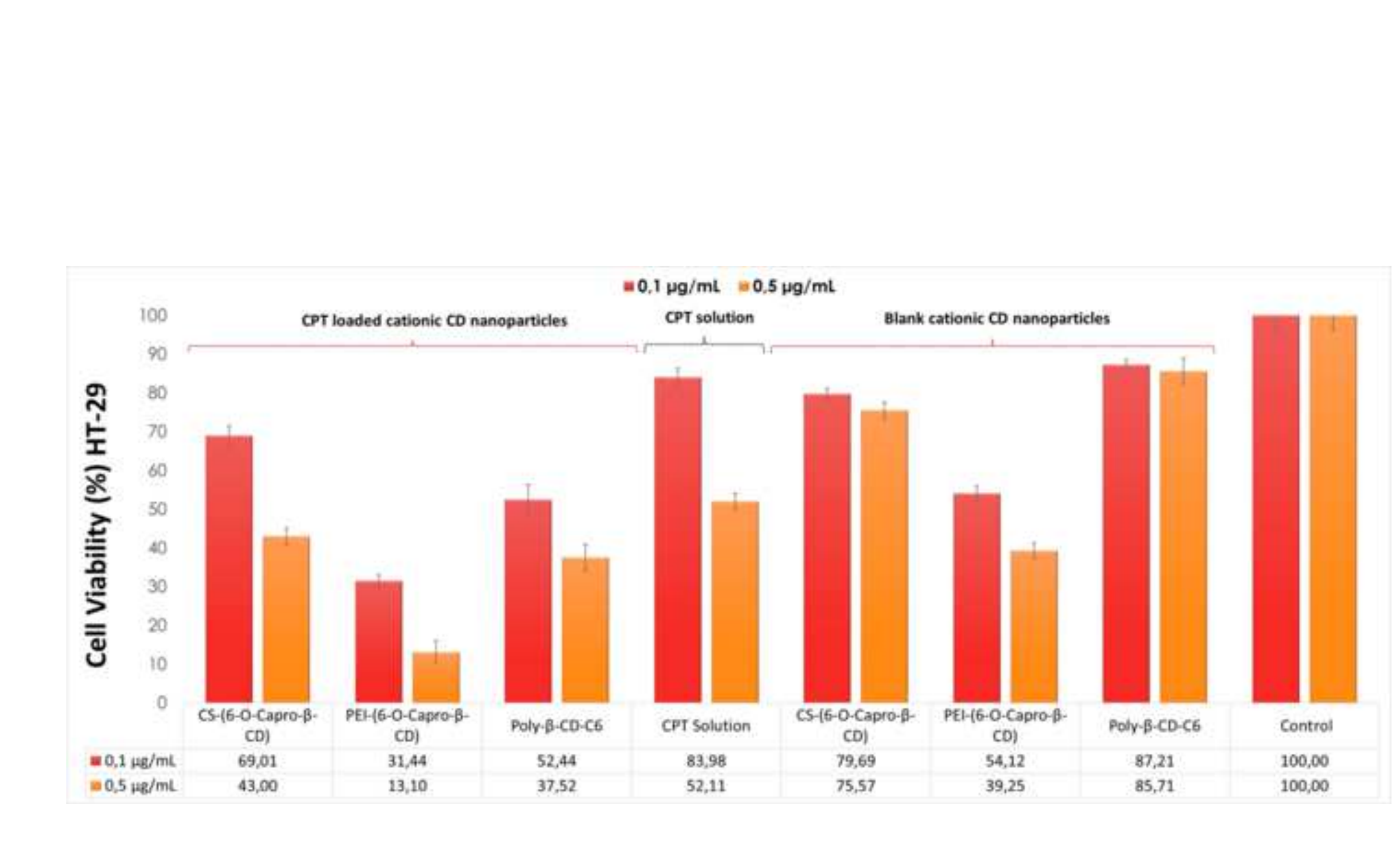

)


Supplementary Material
Click here to download Supplementary Material: SUPPLEMENTARY FILE 6ocaprobcd600.tif

Supplementary Material
Click here to download Supplementary Material: SUPPLEMENTARY FILE 6ocaprobcd600.tif

lick here to download Supplementary Material: SUPPLEMENTARY FILE 6ocaprobcd600.tif

(2)

(1)

probcd600.tif

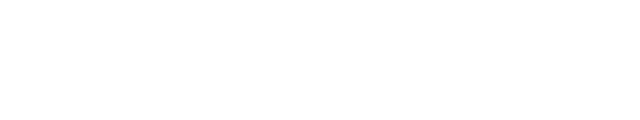
. . . . . . . . . . . . 
Supplementary Material
Click here to download Supplementary Material: SUPPLEMENTARY FILE600.tif

Supplementary Material
Click here to download Supplementary Material: SUPPLEMENTARY FILE600.tif

\begin{abstract}
Click here to download Supplementary Material: SUPPLEMENTARY FILE60.tif
\end{abstract} (1)

Th

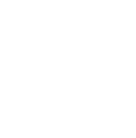
(1) (1)

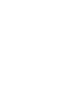
(1)

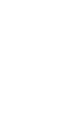

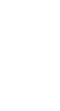

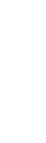
.

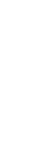

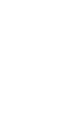

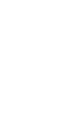
更 更 更

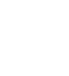
更 (1) 更 (1) 更 (1) 更 .

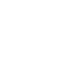


Supplementary Material
Click here to download Supplementary Material: SUPPLEMENTARY FILE2600.tif

Plementary Material: SUPPLEMENTARY FILE2600.tif if

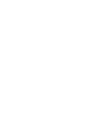

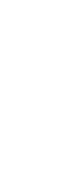
(1)

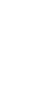

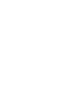
(1) (1) . . . . . . . 西 西 西 西 西 西 西 西 (1) 
Supplementary Material
Click here to download Supplementary Material: Captions for Figures.docx

Supplementary Material
Click here to download Supplementary Material: Captions for Figures.docx

$\mathbf{x}$

pplementary Material: Captions for Figures.docx

(n)

(1)

(1)

(1)

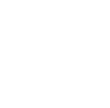

(1)

